\title{
17 Wie steuern automatisierte Fahrzeuge die Raumentwicklung in der Schweiz?
}

Fabienne Perret, Christof Abegg

1. Eine schweizerische Perspektive

2. Die Chancen und Risiken des automatisierten Fahrens

3. Wie automatisierte Fahrzeuge in den Schweizer Markt eindringen könnten

3.1 Drei Szenarien: Generelle Annahmen und spezifische Charakteristik

3.2 Die Szenarien im Vergleich

4. Auswirkungen auf die Raumentwicklung - im Großen wie im Kleinen

4.1 Ein Entwicklungspfad des automatisierten Fahrens

4.4 Räumliche Wirkungen mit Blick auf die drei Szenarien

5. Der Bedarf und die Ansätze eines Regulierungsrahmens für die Schweiz

6. Die Steuerung von Mobilität und Raumentwicklung nicht aus der Hand geben

Fabienne Perret

ETH, Leiterin Geschäftsbereich Verkehr, EBP Schweiz AG

fabienne.perret@ebp.ch

Christof Abegg

Universität Bern, Teamleiter Stadt- und Regionalwirtschaft, EBP Schweiz AG

christof.abegg@ebp.ch 


\section{EINE SCHWEIZERISCHE PERSPEKTIVE}

Die Automatisierung im Verkehr wird unsere Mobilität schrittweise verändern. In dieser Hinsicht ist auch die Schweiz keine Insel in Europa. Forschung, Politik und Praxis setzen sich seit einigen Jahren mit zunehmender Intensität mit dem automatisierten Fahren auseinander. In vielen Fragestellungen laufen die Diskussionen vergleichbar mit anderen Ländern, wenn auch deutlich stärker angetrieben und geprägt von den Unternehmen des öffentlichen Verkehrs als von der Automobilbranche. Eine andere Note erhält die Diskussion auch durch die besondere institutionelle und räumliche Situation der Schweiz. Das ausgeprägt föderalistische System macht einen Dialog über alle Staatsebenen hinweg zur Notwendigkeit. Gefragt ist ein harmonisiertes Zusammenspiel von Bund, Kantonen, Städten und Gemeinden. Hinzu kommt ein komplexes Netz von städtischen Räumen, Agglomerationen, ländlich geprägten Gebieten sowie von Berggebieten - und dies auf vergleichsweise engem Raum.

Vorliegender Beitrag ist eine Synthese aus zwei transdisziplinären Studien, welche die EBP Schweiz AG zusammen mit verschiedenen Partnerinstitutionen in den letzten vier Jahren in der Schweiz durchgeführt hat.

In der ersten Multiclient-Studie wurde zwischen 2016 und 2018 zusammen mit dem Schweizerischen Städteverband, dem BaslerFonds und weiteren Partnern der Einsatz automatisierter Fahrzeuge im Alltag untersucht. Unter dem Obertitel „Denkbare Anwendungen und Effekte in der Schweiz“ wurden ein Grundlagenbericht (EBP, BaslerFonds, Städteverband und weitere Partner 2017), verschiedene Vertiefungsstudien zu spezifischen Fachthemen ${ }^{1}$ (EBP, BaslerFonds, Städteverband und weitere Partner 2018a bis 2018f) sowie ein zusammenfassender Synthesebericht mit Handlungsoptionen für die beteiligten Partner (EBP, BaslerFonds, Städteverband und weitere Partner 2018g) erarbeitet. Die mitfinanzierenden Partner vertraten die öffentliche Hand auf der Ebene von Stadt und Kanton, verschiedene Verkehrsbetriebe und Transportunternehmen sowie eine Versicherung und die nationale Kompetenzzentrale für Verkehrsinformation.

Die zweite Studie wurde unter dem Titel „Automatisiertes Fahren in der Schweiz: Das Steuer aus der Hand geben?" im Auftrag der Schweizerischen Stiftung für Technikfolgenabschätzung (TA-SWISS) ab 2018 erstellt und im Februar 2020 publiziert (Perret et al. 2020). Sie schätzt Chancen und Risiken des vernetzten und automatisierten Fahrens zuhanden von Politik und Gesellschaft ab. Im Sinne einer Auslegeordnung zeigt sie auf, welche Rahmenbedingungen erfüllt werden müssen, damit automatisierte Autos in der Schweiz verkehren können - und wer dafür zuständig sein soll. Die Studie umfasst die Bereiche Politik, Recht, Wirtschaft, Technologie, Gesellschaft und Ethik. Sie nimmt eine Gesamtbeurteilung vor und formuliert Empfehlungen, die an Entscheidungstragende, insbesondere Politikerlnnen, gerichtet sind. Zur breiteren Diffusion der Ergebnisse wurde auch ein Erklärfilm erstellt.

1 Verkehrstechnik, Daten- und IT-Infrastrukturen, mögliche Angebotsformen im kollektiven Verkehr (öffentlicher Verkehr und öffentlicher Individualverkehr), Auswirkungen auf die Verkehrssicherheit, Güterverkehr und Citylogistik auf der Straße, Auswirkungen auf Ressourcen, Umwelt und Klima sowie spezifische Herausforderungen für Städte und Agglomerationen. 
Das Ziel beider Studien bestand nicht darin, die technischen und rechtlichen Fragen in einer möglichst umfassenden Art und Weise zu beantworten. Stattdessen wurde die Technologie so weit beschrieben, dass die wesentlichen Zusammenhänge hervortreten und planerische sowie gesellschaftliche Kernfragen formuliert werden konnten. Aus einer Gesamtbeurteilung wurden Handlungsempfehlungen an Akteure auf allen staatlichen Ebenen, aber auch an Verkehrsunternehmen, Industrie und Branchenverbände abgeleitet. Dabei wurde berücksichtigt, in welchen Bereichen die Schweiz als vergleichsweise kleine Nation überhaupt Einflussmöglichkeiten und Gestaltungsspielraum hat und in welchen Bereichen sie internationale Regelungen übernehmen muss.

Im Folgenden werden wichtige Erkenntnisse aus diesen beiden Studien wiedergegeben und exklusiv für diesen Beitrag in einer Synthese zusammengeführt. Der Artikel verbindet dabei ausgehend von den Chancen und Risiken des automatisierten Fahrens (Abschnitt 2) drei Szenarien zur künftigen Nutzung von automatisierten Fahrzeugen in der Schweiz (Abschnitt 3) mit einer Betrachtung räumlicher Auswirkungen (Abschnitt 4). Der föderalistischen Struktur geschuldet, werden Handlungsbedarf und Handlungsoptionen für den Bund sowie die Städte und Agglomerationen formuliert (Abschnitt 5). Abschließend wird in Abschnitt 6 aufgezeigt, wie das automatisierte Fahren in der Schweiz zu einer zukunftsfähigen Mobilität beitragen kann, wenn alle Staatsebenen entlang des gesamten Politikzyklus und im Zusammenspiel ihren Beitrag leisten.

\section{DIE CHANCEN UND RISIKEN DES AUTOMATISIERTEN FAHRENS}

Das automatisierte Fahren hat das Potential, die Mobilität und den Verkehr von Personen und Gütern grundlegend zu verändern. Bereits heute sind teilautomatisierte Systeme auch in der Schweiz zugelassen und auf dem Markt verfügbar. Deutliche Veränderungen werden aber mit den nächsten Automatisierungsstufen und unter Annahme einer zunehmenden Vernetzung von Fahrzeugen erwartet. Solche hoch- und vollautomatisierten Fahrzeuge werden von Fahrzeugherstellern derzeit entwickelt und sollen je nach Experteneinschätzung ab 2025 bis 2050 zugelassen werden und ihren Durchbruch erfahren. In der internationalen Fachwelt werden die möglichen Effekte des automatisierten und vernetzten Fahrens auf zahlreiche Lebensbereiche breit diskutiert.

Aus heutiger Sicht scheint klar, dass mögliche Nutzungen von automatisierten Fahrzeugen zahlreiche Chancen bieten. Beispielsweise können neue Personengruppen von einer individuellen Mobilität profitieren (Kinder, SeniorInnen), zudem kann die Verkehrssicherheit gesteigert werden. Durch eine erhöhte Effizienz im Betrieb der Infrastrukturen können auch zusätzliche Kapazitäten geschaffen und die Erreichbarkeiten in der Schweiz verbessert werden. Im Weiteren sind neue Möglichkeiten für die Verkehrssteuerung, Flächengewinne in Städten und neue verkehrliche Angebotsformen im öffentlichen Verkehr (ÖV) resp. öffentlichen Individualverkehr (ÖIV) denkbar. Zum letzten Punkt werden in Schweizer Pilotprojekten bereits automatisierte Shuttles getestet (z. B. in den Städten Sion, Fribourg, Meyrin, Neuhausen, Zug und Bern). Auch in der Logistik und im Güterverkehr sind durch die Anwendung automatisierter Systeme große Effizienz- und Attraktivitätsgewinne zu erwarten.

Auf der anderen Seite sind aber auch Risiken absehbar: Besonders relevant ist die potentielle Zunahme der Verkehrs- und Fahrleistung. Vollautomatisierte Fahrzeuge könnten beispielsweise eigenständig zwischen dem Wohn- und dem Arbeitsort oder der Schule verkehren sowie Güter ausliefern. Entsprechende Leerfahrten erhöhen die Fahrleistung. Mit der Nutzung der Reisezeit 
für andere Aktivitäten sinken zudem die persönlichen Reisezeitkosten. Diese Vorteile könnten in längere und häufigere Fahrten reinvestiert werden und damit zu einer weiteren Zersiedelung führen. Zudem wird die wichtige Bedeutung des ÖV im heutigen Sinne durch neue konkurrierende Angebote in Frage gestellt. Die drohende Zunahme der Fahrleistungen auf der Straße widerspricht den Zielen der Schweizer Bundespolitik zur Aufrechterhaltung einer leistungsfähigen Verkehrsinfrastruktur, weil diese häufig nicht weiter ausgebaut werden kann oder soll.

Solche Abschätzungen der Chancen und Risiken für die Schweiz sind insbesondere aus Sicht der staatlichen Akteure von großer Wichtigkeit, da unter anderem auch die Rolle des Staates zu definieren und die Aufgabenteilung zwischen öffentlicher Hand und Privatwirtschaft zu regeln ist. Das Zeitfenster für grundlegende Entscheide ist heute offen, dürfte sich aber hinsichtlich der erwarteten zeitlichen Entwicklung bald schließen. Von diesen Fragestellungen sind zahlreiche Fachgebiete betroffen: Neben rein technologischen und verkehrlichen Fragen ergeben sich auch zahlreiche Fragen zu Wirtschaft, Recht, Politik, Gesellschaft und Ethik.

\section{WIE AUTOMATISIERTE FAHRZEUGE IN DEN SCHWEIZER MARKT EINDRINGEN KÖNNTEN}

Um die mögliche Spannbreite der künftigen Nutzung von automatisierten Fahrzeugen aufzuzeigen, werden in der TA-SWISS-Studie drei Szenarien eingesetzt. Auch wenn die Digitalisierung im Verkehrsbereich derzeit schnell voranschreitet, ist die weitere Entwicklung keineswegs determiniert: Technologische Durchbrüche und politische Weichenstellungen könnten scheinbar gegebene Pfadabhängigkeiten künftig durchaus infrage stellen. Für die vorliegende Studie interessieren in erster Linie spätere Zustände mit vollautomatisierten Fahrzeugen, wenn die LenkerInnen die Kontrolle über das Auto vollständig abgeben kann.

\subsection{DREI SZENARIEN: GENERELLE ANNAHMEN UND SPEZIFISCHE CHARAKTE- RISTIK}

In allen drei Szenarien wird davon ausgegangen, dass allgemeine Megatrends (wie Bevölkerungszunahme, zunehmende Alterung oder Individualisierung) die Entwicklung beeinflussen werden. Je nach Szenario gewinnt allerdings der eine oder andere Trend mehr an Bedeutung oder schreitet schneller voran. Sie unterscheiden sich vor allem in zwei Variablen: Einerseits werden die verschiedenen Räume in der Schweiz differenziert, andererseits wird zwischen einer stark individualisierten und einer kollektiven Nutzung der automatisierten Fahrzeuge unterschieden. Der kollektive Verkehr beschreibt alle Verkehrsformen, bei der eine Person ein nicht privates Fahrzeug nutzt, welches auch von anderen Personen während seiner/ihrer Fahrt zumindest zeitweise - mitgenutzt werden kann, d. h., das Fahrzeug wird simultan geteilt resp. gepoolt (Car-Pooling oder Ride-Sharing).

Im ersten Szenario der stark individualisierten Nutzung herrscht in allen Räumen eine stark individuelle Nutzung vor, die Entwicklung verläuft marktgetrieben und weitgehend ohne Einflussnahme durch die Politik. Automobilhersteller und Datenlieferanten/-verarbeiter sind die wesentlichen Treiber. Nur dort, wo es zu Sicherheitsproblemen oder Engpässen kommt, greift der Staat mit minimalen Regeln ein. 
Im zweiten Szenario mit neuen kollektiven Mobilitätsangeboten in Städten und Agglomerationen nimmt die öffentliche Hand bei der Einführung automatisierter Fahrzeuge eine aktivere Rolle ein. Mittels geeigneter Regulierungen verschafft sie dem kollektiven Verkehr in dichten Räumen Marktvorteile, erlässt Vorschriften über den Datenaustausch und greift steuernd in den Verkehrsfluss ein. Dieser Eingriff soll die Auslastung der Verkehrsnetze in Städten und Agglomerationen siedlungsverträglich lenken und weiterhin eine attraktive Mobilität für alle ermöglichen. Außerhalb dieser Räume, wo die Netzauslastung geringer ist, sieht die Politik keine Notwendigkeit für einen Eingriff und überlässt die Entwicklung den marktwirtschaftlichen Treibern.

Das dritte Szenario, das schweizweit eine stark kollektiv geprägte Nutzung ins Zentrum stellt, bedingt eine sehr aktive und flächendeckende Einflussnahme durch den Staat. Kollektive Verkehrsangebote auch bei geringer Nachfrage sicherzustellen, benötigt entsprechende Abgeltungen und eine konsequente Gesetzgebung. Auch die dazu notwendige schweizweite lückenlose Abdeckung mit zuverlässiger Kommunikationsinfrastruktur ist aufwändig. In diesem Szenario werden Energie- und Umweltziele von der Politik sehr hoch gewichtet.

\subsubsection{Szenario 1: Stark individualisierte Nutzung}

Die stark marktwirtschaftlich getriebene und individualisierte Nutzung automatisierter Privatfahrzeuge führt in Szenario $1 \mathrm{im}$ Personenverkehr zu einer verkehrlichen Verlagerung vom ÖV hin zum motorisierten Individualverkehr (MIV). Zudem dürften Leerfahrten ohne Restriktionen entstehen, beispielsweise aufgrund des Abholens/Bringens von Personen. Auch im Güterverkehr ist eine Verlagerung hin zu kleineren Sendungsgrößen zu erwarten. Die Bündelung der Verkehrsnachfrage nimmt damit sowohl im Personen- als auch im Güterverkehr ab und die Fahrzeugbewegungen steigen in allen Räumen. Abbildung 1 visualisiert die Bewältigung der Verkehrsnachfrage mit den entsprechenden Verkehrsmitteln im Personenverkehr.

Insbesondere in Zentren dürfte die Nachfrage die vorhandenen Verkehrskapazitäten übersteigen, infolgedessen Verkehrszusammenbrüche zu erwarten sind. Dadurch nimmt die Erreichbarkeit der Zentren ab, was zu einem erhöhten Druck auf Agglomerationen und auf periphere Gebiete führt. Die Gesamtverkehrsleistung in der Schweiz steigt durch verlängerte Wege infolge der räumlichen (Um-)Verteilung. Es dürfte zu einer größeren Zersiedelung kommen, wenn die Raumplanung nicht steuernd eingreift. Hierzu gibt die andere Studie im zweiten Teil des Beitrags weitere Hinweise.

Durch die geringe Bündelung der Verkehrsnachfrage steigt auch der Bedarf an Infrastrukturen sowie Fahrzeugen und folglich Energie, infolgedessen mit erhöhten Emissionen zu rechnen ist. Da der Staat die Vernetzung der Fahrzeuge untereinander vorwiegend den Herstellern und Datenlieferanten überlässt, muss er keine aufwändigen öffentlichen Dateninfrastrukturen realisieren. Die Übersicht aller Auswirkungen ist der später folgenden Abbildung 4 zu entnehmen.

\subsubsection{Szenario 2: Neue Angebote in Städten und Agglomerationen}

Durch die neuen Mobilitätsangebote erhält der kollektive Verkehr eine große Bedeutung in dichten Räumen. Für diese Räume sind im Personenverkehr Abnahmen beim MIV zu erwarten. In peripheren Gebieten hat der Kollektivverkehr hingegen eine geringe Bedeutung. Zudem kommen dort Leerfahrten dazu, v. a. durch das Abholen/Bringen von Personen oder Gütern. Während also die Bündelung in den Städten und Agglomerationen eher zunimmt, nimmt sie in weniger dichten Räumen hingegen ab. Schweizweit betrachtet könnten sich die zunehmenden 
und abnehmenden Fahrzeugbewegungen in etwa ausgleichen. Abbildung 2 visualisiert die Bewältigung der Verkehrsnachfrage mit den entsprechenden Verkehrsmitteln im Personenverkehr. Analoge Wirkungen sind auch beim Güterverkehr zu erwarten.

Durch die hoheitliche Verkehrsbeeinflussung in Städten und Agglomerationen werden dort Nachfrage und Angebot aufeinander ausgerichtet. Die Erreichbarkeiten bleiben gegenüber heute ungefähr konstant oder verbessern sich gar leicht. Da die Reisezeit in automatisierten Fahrzeugen mit anderen Aktivitäten genutzt werden kann, gewinnen periphere Räume als Wohnstandorte an Attraktivität. Die Gesamtverkehrsleistung in der Schweiz steigt durch verlängerte Wege - im Szenario 2 gibt es solche allerdings nur außerhalb der Agglomerationen, weswegen die gesamte Zunahme etwas geringer ausfallen wird als im Szenario 1.

Der Bedarf an Fahrzeugen und Energie verändert sich vorerst nicht maßgebend. Erst wenn es bei einer hohen Durchdringung gelingt, in den Städten und Agglomerationen viele Verkehrsteilnehmende vom MIV auf den ÖIV zu bringen, könnte der Bedarf an Fahrzeugen sinken. Mit weniger Fahrzeugen könnten dann die gleichen Personenbewegungen sichergestellt werden, woraufhin sich der Energiebedarf für die Herstellung der Fahrzeuge sowie deren Betriebsenergie und die zu erwartenden Emissionen mindert. Mit dem Verkehrsmanagement in Städten und Agglomerationen wird die bestehende Infrastruktur möglichst effizient genutzt, sodass bedeutende Ausbauten nicht notwendig sind. Hierfür muss die öffentliche Hand die Vernetzung sicherstellen. Allenfalls dürften sich infolge einer bedeutenden Nutzung von Sammeltaxis die benötigten Parkflächen in dichten Räumen etwas reduzieren. In den peripheren Gebieten sind lokal Ausbauten der Verkehrsinfrastruktur zur Bewältigung der erhöhten Verkehrsnachfrage denkbar. Die Übersicht aller Auswirkungen ist der Abbildung $4 \mathrm{zu}$ entnehmen.

\subsubsection{Szenario 3: Schweizweit stark kollektiv geprägter Verkehr}

Die große Bedeutung des kollektiven Verkehrs geht mit deutlichen Abnahmen der individuelIen Mobilität in allen Räumen einher. Leerfahrten, die zwischen Fahrten mit NutzerInnen unterwegs sind, treten nur in begrenztem Rahmen und bei Sammeltaxis auf. Die Bündelung ist allgemein hoch, sodass eine deutlich geringere Anzahl an Fahrzeugbewegungen ausreicht, um die Verkehrsnachfrage zu befriedigen. Abbildung 3 visualisiert die Bewältigung der Verkehrsnachfrage mit den entsprechenden Verkehrsmitteln im Personenverkehr. Analoge Wirkungen sind auch beim Güterverkehr zu erwarten.

Die Nachfrage wird durch die hoheitliche Verkehrssteuerung aktiv beeinflusst und auf das Angebot abgestimmt. Da in peripheren Räumen weniger Sammeltaxis verkehren, können Wartezeiten auftreten, was die Erreichbarkeit verschlechtert. Zudem könnten die Preise für die Mobilität steigen, weil die Aufrechterhaltung eines öffentlichen Angebots kostenintensiv ist. Die Verkehrsleistung würde bei hoher Durchdringung schweizweit sinken.

Der Bedarf an Fahrzeugen wird deutlich geringer. Durch eine verringerte Gesamtverkehrsleistung nehmen zudem der Energiebedarf sowohl für Herstellung als auch Betrieb und die zu erwartenden Emissionen ab. Mit dem Verkehrsmanagement in allen Räumen wird die bestehende Infrastruktur möglichst effizient genutzt, Ausbauten sind keine nötig. Dafür muss die öffentliche Hand die Vernetzung intensiv sichern. Infolge einer bedeutenden Nutzung des kollektiven Verkehrs mindert sich der Bedarf an Parkflächen in allen Räumen deutlich. Die Übersicht aller Auswirkungen ist der Abbildung 4 zu entnehmen. 
Abbildung 1: Visualisierung der verkehrlichen Auswirkungen im Szenario 1. Die Anzahl der dargestellten Fahrzeuge visualisiert die Höhe des Verkehrsaufkommens im jeweiligen Szenario für eine gleichbleibende Anzahl Verkehrsteilnehmender.

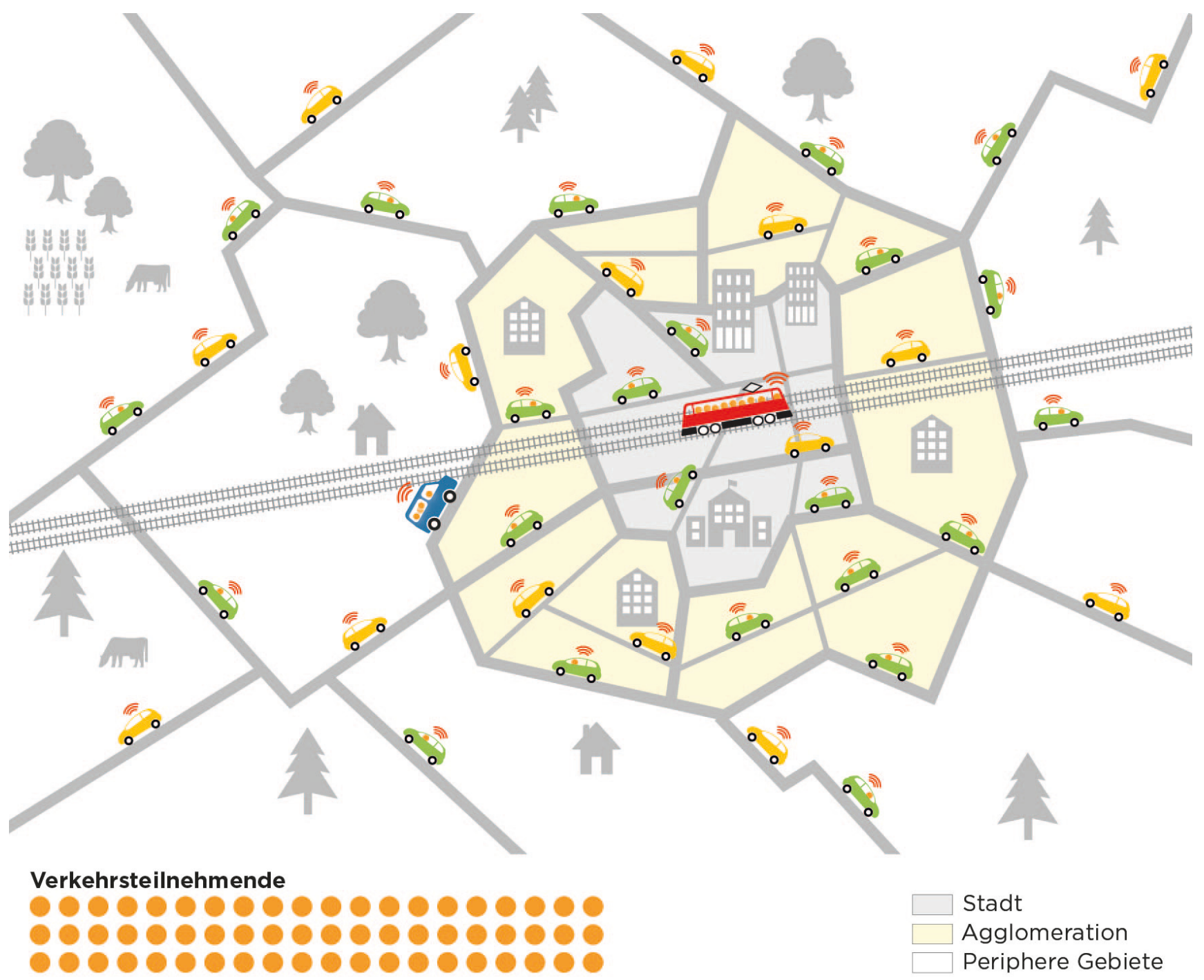

MIV: Belegte Fahrzeuge

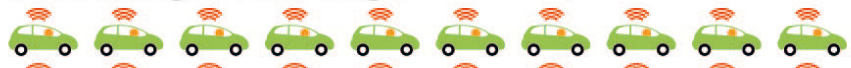

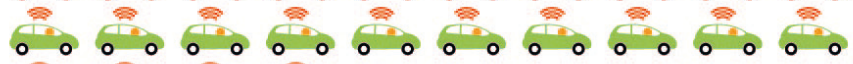

$\therefore 000000$

\section{MIV: Leerfahrten}

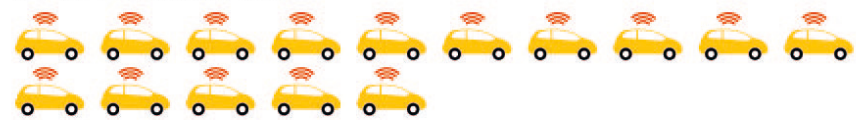

\section{öIV (Shuttle)}

\section{ڤ.}

\section{öv}


Abbildung 2: Visualisierung der verkehrlichen Auswirkungen im Szenario 2. Die Anzahl der dargestellten Fahrzeuge visualisiert die Höhe des Aufkommens im jeweiligen Szenario für eine gleichbleibende Anzahl Verkehrsteilnehmender.

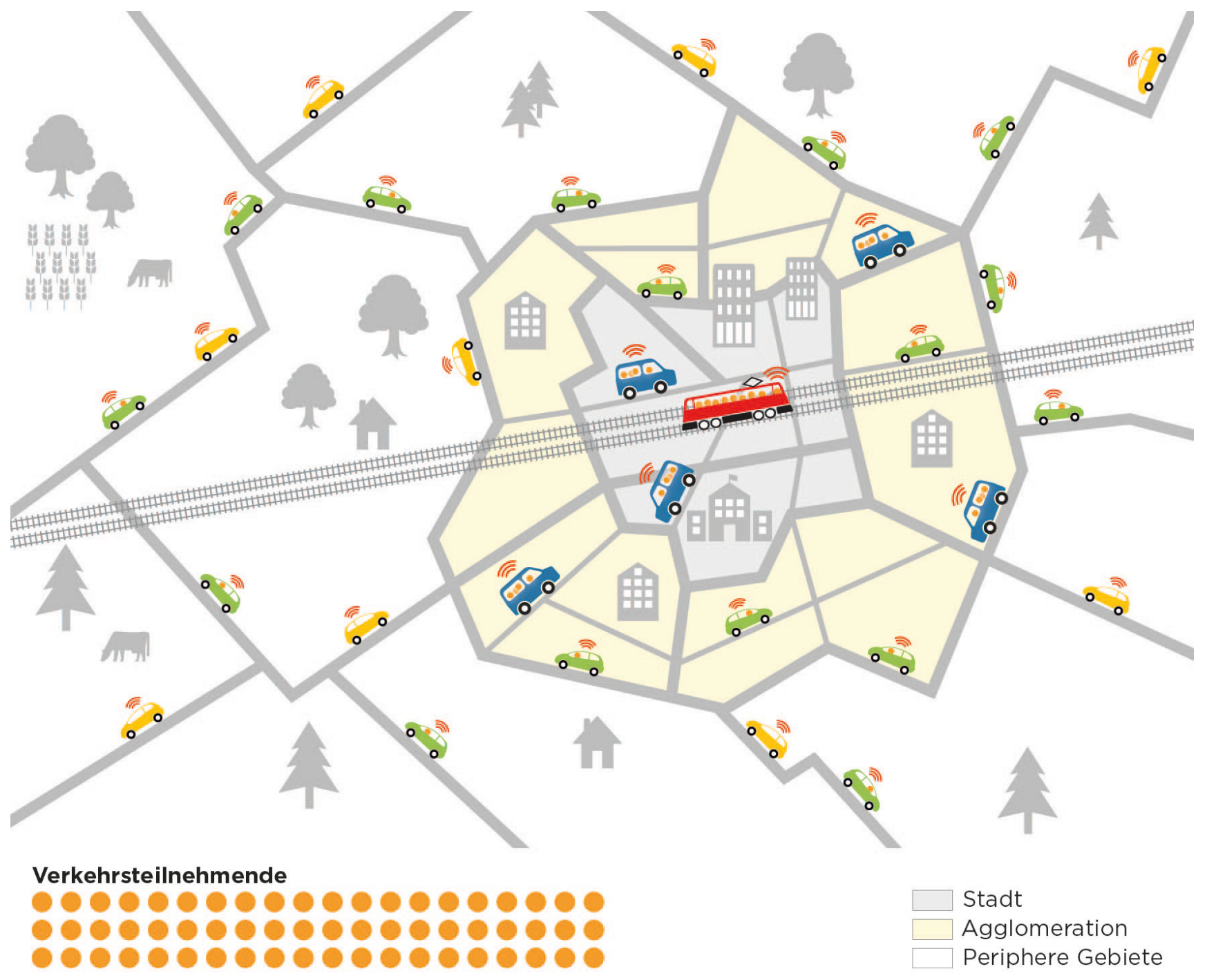

MIV: Belegte Fahrzeuge

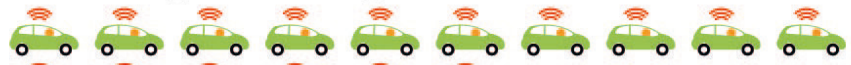

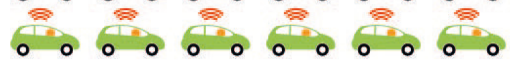

\section{MIV: Leerfahrten}

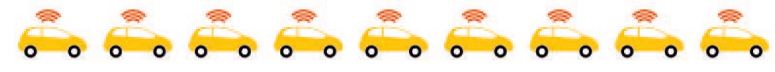

ÖIV (Shuttle)

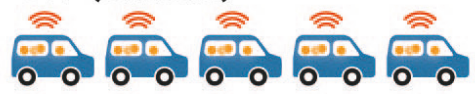

öV

Thanmin

Quelle: Perret et al. (2020) 
Abbildung 3: Visualisierung der verkehrlichen Auswirkungen in Szenario 3. Die Anzahl der dargestellten Fahrzeuge visualisiert die Höhe des Aufkommens im jeweiligen Szenario für eine gleichbleibende Anzahl Verkehrsteilnehmender.

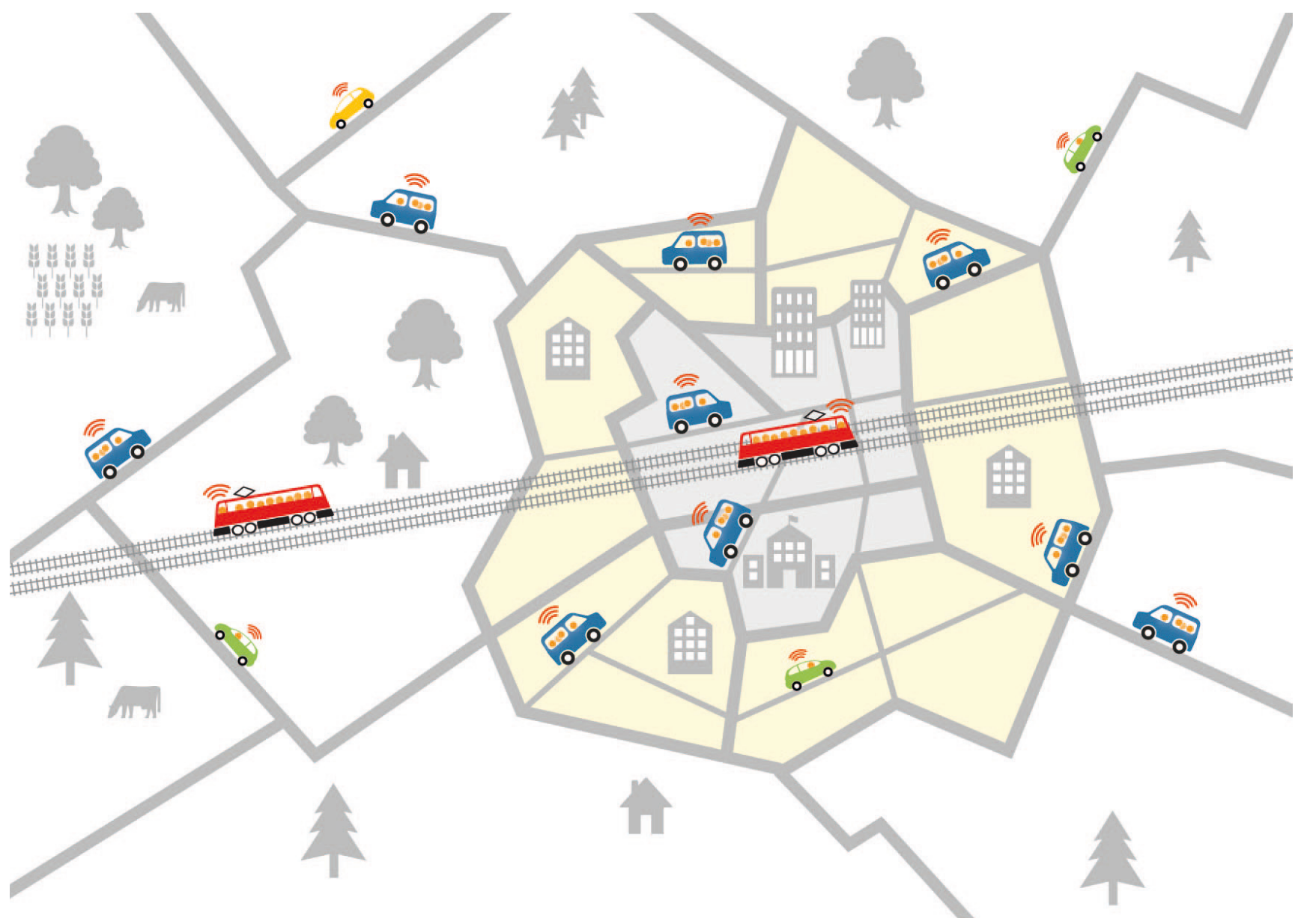

Verkehrsteilnehmende

00000000000000000000

O

00000000000000000000

Stadt

Agglomeration

Periphere Gebiete

\section{MIV: Belegte Fahrzeuge}

$\therefore 00000$

\section{MIV: Leerfahrten}

$\therefore 0$

\section{ÖIV (Shuttle)}

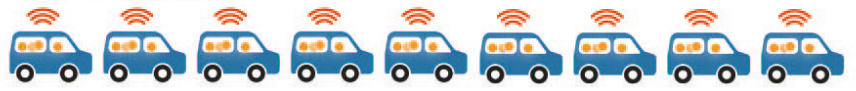

\section{öv} 玄

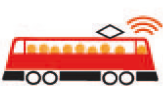




\subsection{DIE SZENARIEN IM VERGLEICH}

Die folgende Abbildung 4 stellt die geschilderten qualitativen Auswirkungen der drei Nutzungsszenarien einander gegenüber.

Abbildung 4: Übersicht der qualitativen Auswirkungen in allen Szenarien

\begin{tabular}{|c|c|c|c|}
\hline $\begin{array}{l}\text { Starke Erhöhung } \\
\text { Leichte Erhöhung } \\
\text { Gleichbleibend } \\
\text { Leichte Reduktion } \\
\text { Starke Reduktion }\end{array}$ & $\begin{array}{c}\text { Szenario } 1 \\
\text { Stark individualisierte } \\
\text { Nutzung }\end{array}$ & $\begin{array}{c}\text { Szenario } 2 \\
\text { Neue Angebote } \\
\text { in Städten und } \\
\text { Agglomerationen }\end{array}$ & $\begin{array}{c}\text { Szenario } 3 \\
\text { Schweizweit stark } \\
\text { kollektiv geprägter } \\
\text { Verkehr }\end{array}$ \\
\hline \multicolumn{4}{|c|}{ Verkehrsleistung Personen } \\
\hline \multicolumn{4}{|c|}{ Anteil kollektive Mobilität } \\
\hline \multicolumn{4}{|l|}{ Fahrleistung MIV } \\
\hline \multicolumn{4}{|c|}{ Transportleistung Güter } \\
\hline \multicolumn{4}{|l|}{ Anteil Schiene } \\
\hline \multicolumn{4}{|c|}{ Fahrleistung Güter auf Straße } \\
\hline \multicolumn{4}{|c|}{ Räumliche Konzentration } \\
\hline \multicolumn{4}{|c|}{ Energie-/Ressourcenverbrauch } \\
\hline \multicolumn{4}{|l|}{ Flächenbedarf } \\
\hline \multicolumn{4}{|l|}{ Datenbedarf } \\
\hline Verkehrssicherheit & & & \\
\hline
\end{tabular}

Quelle: Perret et al. (2020)

Während Szenario 1 voraussichtlich zu mehr Verkehrs- und Fahrleistung im Güter- und Personenverkehr führt, könnte in Szenario 3 die Fahrleistung deutlich sinken, ohne dass die Bevölkerung auf Mobilität resp. die Logistik für Gütertransporte verzichten muss. In Szenario 2 heben sich tendenzielle Zunahmen im ländlichen Raum und Abnahmen in Agglomerationen schweizweit in etwa auf. Am deutlichsten zeigt sich dieser Unterschied beim Ressourcenbedarf: In Szenario 1 könnte dieser in Bezug auf Energie und Flächen deutlich steigen, während in Szenario 3 bei zunehmender Bündelung von Fahrten eine deutliche Reduktion möglich wäre. Der Datenbedarf hingegen nimmt bei allen Szenarien zu. 


\section{AUSWIRKUNGEN AUF DIE RAUMENTWICKLUNG - IM GROSSEN WIE IM KLEINEN}

\subsection{EIN ENTWICKLUNGSPFAD DES AUTOMATISIERTEN FAHRENS}

Während die TA-SWISS-Studie drei Szenarien formuliert hat, wurde im Forschungsprojekt „Einsatz automatisierter Fahrzeuge im Alltag - Denkbare Anwendungen und Effekte in der Schweiz" ein alternativer Zugang gewählt. Hier wurde eine Storyline des Einsatzes von automatisierten Fahrzeugen in der Schweiz entwickelt. Dabei handelt es sich um einen Entwicklungspfad, der aus fachlicher Sicht in der Schweiz als plausibel eingeschätzt wird. Die Storyline erhebt jedoch keineswegs den Anspruch, die einzig mögliche Entwicklung darzustellen.

Die Storyline ist als eine Abfolge von Zuständen definiert, die sich durch Übergänge mit ändernden Voraussetzungen abgrenzen (Abb. 5). Der jeweils folgende Zustand beginnt, wenn alle Voraussetzungen erfüllt sind, die unter anderem rechtliche Aspekte (Zulassungen, Versicherungen, Straßenrecht), technologische Meilensteine, Veränderungen in der Infrastruktur und die gesellschaftliche Akzeptanz betreffen. Für die Studie wurden sechs Zustände definiert.

Abbildung 5: Illustration der Storyline

Anteil automatisierter Fahrleistung

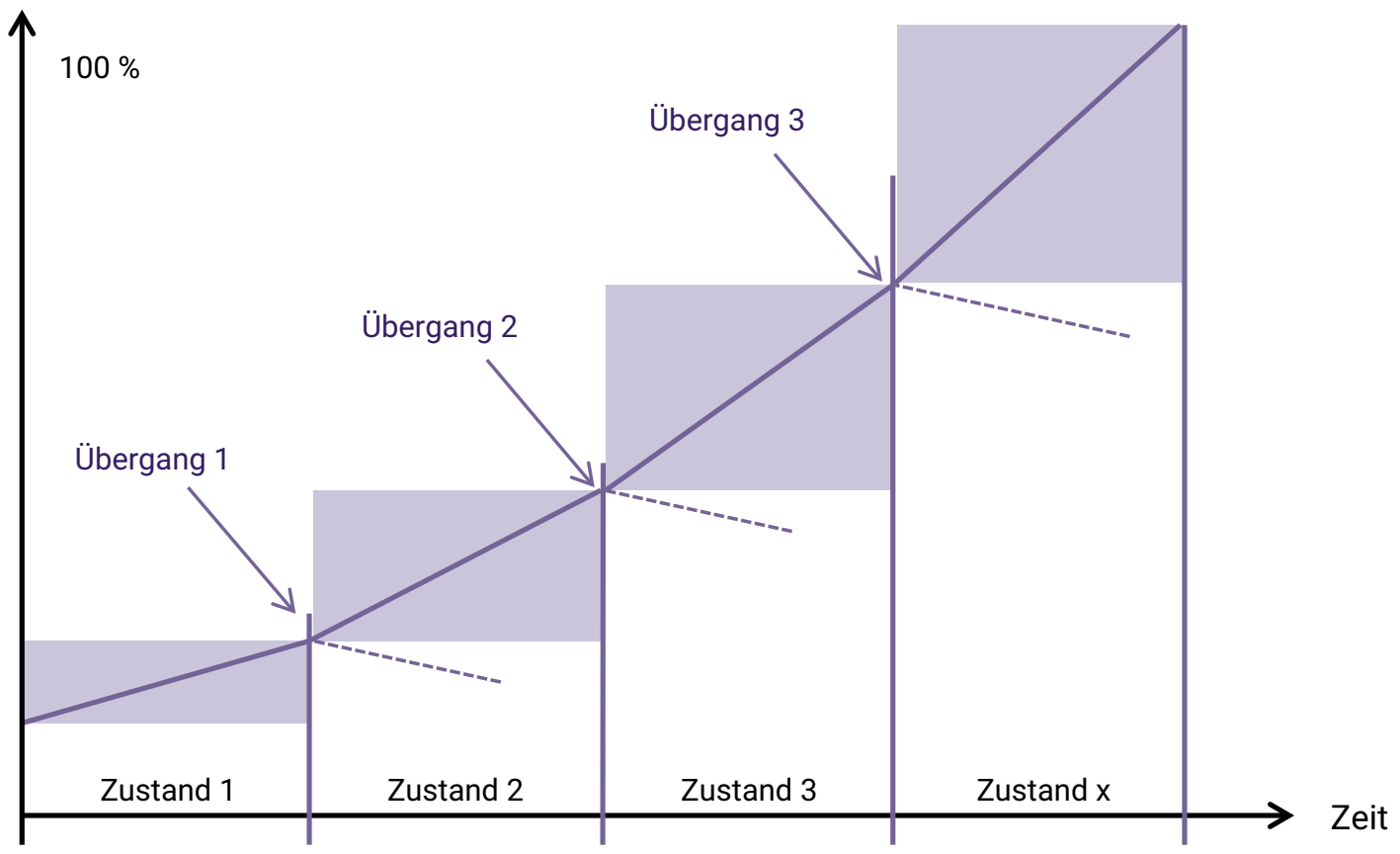

Quelle: EBP/BaslerFonds/Städteverband und weitere Partner ( 2017)

Mit der Storyline wird die Komplexität reduziert, damit die relevanten Aspekte für das Handeln der Akteure herausgeschält werden können. Sie liefert damit eine geeignete Grundlage für weiterführende und vertiefende Studien. Zudem ergeben sich aus der Storyline in sich schlüssige Zustände, die eine Auseinandersetzung mit den Auswirkungen des automatisierten Fahrens auf verschiedenen staatlichen und räumlichen Ebenen ermöglichen. 
Im Rahmen der Gesamtstudie hat das Teilprojekt „Städte und Agglomerationen“ die Auswirkungen des Einsatzes automatisierter Fahrzeuge auf die Siedlungsräume in der Schweiz analysiert. Automatisiertes Fahren löst Effekte auf zwei räumlichen Maßstabsebenen aus. Im Zentrum der Studie stehen kleinräumige Auswirkungen in dichteren Städten und Agglomerationen. Ergänzend wurden mögliche großräumige Auswirkungen auf die Siedlungsstruktur beleuchtet.

\subsection{GROSSRÄUMIGE AUSWIRKUNGEN AUF DIE SIEDLUNGSSTRUKTUR}

Die Raumstruktur dürfte insbesondere durch die Erreichbarkeitsveränderungen im MIV maßgeblich verändert werden. Dabei werden die ländlichen Räume am meisten profitieren: Kapazitätssteigerungen ermöglichen substantielle Erreichbarkeitsgewinne, die Nutzung der Reisezeit wird die Attraktivität zum Pendeln in zentrale Räume weiter erhöhen. Gerade in ländlichen Räumen mit reduzierten ÖV-Angeboten werden bisher weniger mobile Bevölkerungsgruppen wie Kinder oder ältere Menschen ihre Mobilität verbessern können.

In Städten und Agglomerationen muss hingegen mit geringeren oder gar negativen Erreichbarkeitsgewinnen gerechnet werden, weil aufgrund des erwarteten Mehrverkehrs sowie der beschränkten Kapazitäten die Überlastung zunehmen könnten. Hinzu kommt die Gefahr, dass Mehrverkehr und die dicht fahrenden Fahrzeuge die Wohn- und Aufenthaltsqualität in städtischen Räumen reduzieren.

Aufgrund der relativen Attraktivitätsverschiebung muss einerseits im ländlichen Raum mit einer anhaltenden bzw. noch verstärkten Tendenz zur Zersiedlung gerechnet werden. Andererseits ist aber auch zu erwarten, dass Effizienzgewinne im ÖV und neue Angebotsformen im ÖIV die Attraktivität städtischer Räume erhöhen und bestehende Urbanisierungstendenzen je nach Szenario weiter verstärken können. Für die raumstrukturellen Wirkungen wird das Zusammenspiel bzw. die Konkurrenz zwischen MIV, ÖV und ÖIV bedeutsam sein. Die automatisierte Mobilität allein wird das Verhältnis der Räume kaum grundlegend verändern. Andere Entwicklungen, wie demographische Veränderungen, Digitalisierung und veränderte Wertschöpfungsketten oder klimabedingte Einflüsse werden die raumstrukturelle Entwicklung mindestens so stark prägen.

\subsection{AUSWIRKUNGEN IN STÄDTEN UND AGGLOMERATIONEN}

Die Wirkungen des automatisierten Fahrens für Städte und Agglomerationen werden für folgende Verkehrsarten differenziert beschrieben: fließender Verkehr, ruhender Verkehr, ÖV, ÖIV, Fuß- und Radverkehr sowie Güterverkehr. Die möglichen Effekte des automatisierten Fahrens im Siedlungsraum fallen je nach Funktion einer Straße unterschiedlich aus. Sie sind abhängig vom jeweiligen Kontext mit den angrenzenden Nutzungen und einer "gelebten Mobilität“. Deshalb wurden in der Studie für eine räumlich differenzierte Betrachtung folgende „Mobilitätsräume" unterschieden:

- Hauptverkehrsstraßen im Siedlungsraum („Hauptverkehrsstraße“)

- Straßen in Orts- und städtischen Quartierzentren („Zentrumsstraße“)

- Erschließungsstraßen in Wohnquartieren („Quartierstraße“)

- Erschließungsstraßen im Gewerbegebiet („Gewerbegebiet“)

- multimodale Verkehrshubs im Siedlungsgebiet („Verkehrshub“) 


\section{Fließender Verkehr}

Durch neue Angebote, mögliche Leerfahrten und neue Nutzergruppen (ältere Menschen, Kinder) wird die motorisierte Fahrleistung gegenüber heute zunehmen. Können einerseits die Zeitlücken zwischen den Fahrzeugen infolge der Automatisierung reduziert werden, stellen sich andererseits Kapazitätsgewinne bei der bestehenden Infrastruktur ein. Die Zunahme verhält sich überproportional zur Durchdringung der Gesamtflotte mit automatisierten Fahrzeugen. Einzelne Straßen, wie beispielsweise die Hauptverkehrsstraßen, können durch die Automatisierung ihre Kapazitäten erhöhen, andere wie die Zentrumsstraßen eher nicht. Es kommt zu einer Um- und Ungleichverteilung des Mehrverkehrs. Insgesamt ist davon auszugehen, dass der Mehrverkehr die Kapazitätssteigerung in allen Mobilitätsräumen überkompensiert und spürbar wird. Die Kapazitätseffekte können eventuell helfen, vereinzelt Infrastrukturausbauten nicht oder später notwendig werden zu lassen.

Ein Eigentrasse für automatisierte Fahrzeuge in Siedlungsräumen scheint aus heutiger Sicht in der Schweiz wenig realistisch, da meist kein Platz und eine hohe Knotendichte vorhanden sind. Solange sich eine bedeutende Anzahl menschlich gesteuerter Fahrzeuge auf den Straßen bewegen und sich die Fahrzeuggrößen der maßgebenden Nutzungen (bspw. ÖV, Lkw, Rettungsdienste, Winterdienst) nicht verändern, erscheint eine Reduktion der Fahrbahnbreiten wenig realistisch.

Auf Hauptverkehrsstraßen wirken sich die Effekte des automatisierten Fahrens überwiegend positiv aus. Insgesamt zeichnet sich hier der Straßenraum durch eine geringere Komplexität aus als beispielsweise in Zentren mit einem Nebeneinander von unterschiedlichsten Verkehrsteilnehmenden. Viele der angrenzenden Nutzungen können nicht direkt über die Hauptverkehrsstraße erreicht werden. Dabei ergeben sich Chancen für den Verkehrsfluss, der sich mit einem hohen Anteil an automatisierten und vernetzten Fahrzeugen verbessern dürfte, da die Fahrzeuge dichter und mit konstanteren Geschwindigkeiten verkehren. Durch die Verkehrszunahme und den Umstand, dass automatisierte Fahrzeuge mit kürzeren Abständen fahren können, wird jedoch auch die Trennwirkung verstärkt.

Im Weiteren können mit adaptiven Spurzuteilungen die Verkehrskapazitäten, welche aufgrund des zeitlich variierenden Pendlerverkehrs oftmals monodirektional sind, erhöht werden. Auch bei Knoten werden komplexere und adaptiv geschaltete Grünphasen möglich. Ebenso lässt sich der MIV situativ steuern und somit effizienter über die Knoten führen. Im Stadt- und Agglomerationsgebiet bieten sich Hauptverkehrsstraßen deshalb an, Mehrverkehr aufzunehmen, da durch die Automatisierung auf diesen Straßen die größten Kapazitätsgewinne erzielt werden können. Eine solche Kapazitätserhöhung kann aber einerseits andere Verkehrsteilnehmende einschränken oder dazu führen, dass ihnen die Straßennutzung komplett untersagt wird. Kapazitätsgewinne können andererseits aber auch für den ÖV genutzt werden. Um adaptive Spurführungen und Knotensteuerungen zu ermöglichen, braucht es einen hohen Organisationsaufwand und eine digitale Kommunikationsinfrastruktur. Ein Ausfall der Systeme benötigt eine Rückfallebene zur Sicherstellung der Funktionalität des Straßennetzes, das wiederum noch über lange Zeit mit der notwendigen analogen Ausrüstung sowie der betrieblichen Flexibilität betrieben und unterhalten werden müsste.

Straßen in Zentrumsgebieten haben neben verkehrlichen Funktionen auch eine wichtige Funktion für die Siedlung. Hierbei ist die Wirkung des Mehrverkehrs besonders von Bedeutung, der durch die Parkvorgänge und Güterumschläge und die zu erwartenden Leerfahrten stark beeinflusst wird. Potentielle Kapazitätsgewinne durch die Automatisierung dürften durch die Zunahme der Fahrten und Umschlagvorgänge kompensiert werden. Der Mehrverkehr und die daraus resultierende verstärkte Trennwirkung behindern den ÖV, schränken flächige Querungsmöglichkeiten beispielsweise für Zufußgehende stark ein, beeinträchtigen den Radverkehr und reduzieren die Aufenthaltsqualität. 


\section{Ruhender Verkehr}

Parkplätze verlieren an Bedeutung. Sharing- und Pooling-Angebote sowie die Möglichkeit zu Leerfahrten führen zu einem geringeren Bedarf an (dispersen) Parkplätzen. Zudem können automatisierte Fahrzeuge nach Gebrauch an einem ferneren Standort abgestellt werden, weshalb das Parken nicht mehr gleichermaßen standortgebunden ist. Dadurch wird aber auch Mehrverkehr generiert: Leerfahrten und Fahrten zu weiter weg liegenden Parkplätzen erhöhen die Fahrleistung auf dem umliegenden Straßennetz. Zudem werden designierte Halteflächen außerhalb der Fahrbahn für das Ein- und Aussteigenlassen bei On-demand- und Pooling-Angeboten sowie bei automatisierten Privatfahrzeugen benötigt.

Zentrumsstraßen weisen eine hohe Nutzungswechselfrequenz mit häufigen Zu- und Wegfahrten auf, wobei Parkplätze dazu dienen, die umliegenden Nutzungen zu erreichen. Parkmöglichkeiten am Straßenrand können unter Umständen größtenteils wegfallen und/oder durch Umschlagsflächen ersetzt werden. Das Parken kann auf neuen und umgenutzten zentralisierten Parkflächen konzentriert werden, wobei die "gewonnenen“ Flächen für verschiedene anderweitige Nutzungen umgewidmet werden können. Je nach Situation erlaubt dies einen neuen Radfahrstreifen, breitere Gehsteige oder zusätzliche Grünelemente für die Straßenraumgestaltung. Im Gegenzug werden für ein vollautomatisiertes „Abladen und Abholenlassen“ von Personen und Gütern markierte Halteflächen notwendig, deren Benutzung es zu regeln gilt.

In Wohnquartieren ist die Zu- und Wegfahrfrequenz deutlich geringer als bei Zentrumsstraßen, der Parkplatz dient dem Abstellen der Fahrzeuge während nicht benötigten Zeiten. Dank reduziertem individuellem Fahrzeugbesitz und der Verlagerung von Parkplätzen auf zentrale Parkflächen kann der Straßenraum in Wohnquartieren für den Fuß- und Radfahrverkehr attraktiver gestaltet und mehr Platz für das Quartierleben gewonnen werden. Das Anhalten auf Quartierstraßen erfolgt im Straßenraum oder auf privaten Vorplätzen, was wiederum Platz beansprucht, jedoch ohne, dass dies gesteuert werden muss.

\section{ÖV und ÖIV}

Es ist davon auszugehen, dass konventionelle und gebündelte Linienangebote mit zunehmend automatisierten Zügen, Trams und großen Bussen weiterhin das Grundgerüst des ÖV-Systems in Städten und Agglomerationen bilden werden. Die Automatisierung, die Vernetzung und neue (digitale) Mobilitätsdienste eröffnen dabei ein erhebliches Rationalisierungspotential, um mehr und attraktivere ÖV-Angebote $v$. a. bei geringerem Passagieraufkommen günstiger produzieren zu können. Neue ÖIV-Angebotsformen werden das ÖV-System daher insbesondere im peripheren Nahverkehrsbereich ergänzen. Dabei wird es sich vor allem um kleine bis mittlere Fahrzeuge handeln, die teilweise oder vollumfänglich nach Bedarf verkehren werden. Besonders von Bedeutung ist das Aufkommen neuer Dienste öffentlicher oder privater Mobilitätsanbieter, welche ein individuelles Abholen „vor der Haustür“ ermöglichen - ohne fixe Haltepunkte und kurzen Wartezeiten. Mit dem zunehmenden Einfluss des adaptiven ÖIV nimmt auch der Bedarf nach zusätzlichen Haltebereichen zu. Noch nicht abschätzbar ist, inwieweit bei solchen Haltebereichen eine Barrierefreiheit sichergestellt werden kann oder muss. Bezeichnete Halteflächen und bestehende ÖV-Haltestellen können zusätzlich mit diesen automatisierten Fahrzeugen angefahren werden. Die Möglichkeiten einer zusätzlichen Nutzung dürften jedoch wesentlich von den Frequenzen solcher Dienste abhängig sein.

Mit der Automatisierung ergeben sich zusätzliche Potentiale bei multimodalen Verkehrshubs. Hier können insbesondere zu Randzeiten bestehende ÖV-Fahrspuren und Haltestellen vielfältiger genutzt werden und so weitere Angebote und dichtere Anschlussmöglichkeiten schaffen. Im gesamten ÖV-Netz bietet die Automatisierung zudem die Möglichkeit, klassische, stark belastete multimodale Verkehrshubs zu entlasten, indem vermehrt tangentiale Verbindungen mittels On-Demand-Services geschaffen werden. Spezifische Fragen ergeben sich auch aus 
einer Automatisierung des Trambetriebs, der vermehrt auf Eigentrassen erfolgen dürfte, was gegenüber dem Mischverkehr einen erhöhten Platzbedarf nach sich zieht. An multimodalen Verkehrshubs kann dies die Durchlässigkeit für den querenden Fuß- und Radverkehr (weiter) beeinträchtigen.

\section{Fuß- und Radverkehr}

Heute wird das Nebeneinander von verschiedenen Verkehrsteilnehmenden vor allem in Zentren und Wohnquartieren erfolgreich gelebt und gefördert, was insbesondere dem Fuß- und Radverkehr entgegenkommt. Sicherheits- und Kapazitätsüberlegungen können bei zunehmender Automatisierung dazu führen, dass Querungsmöglichkeiten zugunsten von Fahrspuren für automatisierte Fahrzeuge eingeschränkt werden. Dadurch würden Mischverkehrslösungen beeinträchtigt und der Fuß- und Radverkehr den Bedürfnissen des automatisierten Fahrens untergeordnet werden. Die Frage der Interaktion von automatisierten Fahrzeugen mit dem Fuß- und Radverkehr sowie mit nichtautomatisierten Fahrzeugen ist noch weitgehend offen. Ein großer Teil der Kommunikation zwischen Verkehrsteilnehmenden erfolgt durch Zeichen und Blickaustausch, was bei vollautomatisierten Fahrzeugen nicht mehr in gleicher Art möglich ist. Es ist ebenfalls zu erwarten, dass Zufußgehende und Radfahrende sich künftig gegenüber automatisierten Fahrzeugen anders verhalten werden.

Die langen Übergangszeiten mit Mischverkehr von automatisierten und nichtautomatisierten Fahrzeugen lassen vermuten, dass bestehende Systeme der Markierung, Signalisation und Steuerung noch längerfristig verwendet werden. Die langen Zeiträume mit Mischverkehr können auch dazu führen, dass sich automatisierte Fahrzeuge auf Mischverkehrsflächen reaktiv, auf „Nummer sicher" gehend, unterordnen werden, womit wiederum der erwartete Nutzen in Bezug auf Kapazitätssteigerung und Fahrfluss deutlich reduziert werden könnte. Notwendig wird ein Ausgleich zwischen dem Erreichen der optimalen Nutzung der Automatisierung und der Fuß- und Radverkehrsqualität bzw. -sicherheit.

Die Wirkungen auf den Fuß- und Radverkehr dürften in allen Mobilitätsräumen spürbar werden. Im städtischen Gesamtsystem wird dadurch die Schaffung bzw. Aufrechterhaltung eines durchgängigen Fuß- und insbesondere Radnetzes anspruchsvoller.

In Orts- und Quartierzentren wirkt sich eine Beeinträchtigung flächiger Querungsmöglichkeiten negativ auf die Qualität des Fuß- und Radverkehrs aus. Gerade hier ist die Durchlässigkeit und Bewegungsfreiheit aufgrund der vielen Nutzungsangebote auf kleinem Raum von großer Bedeutung. Eine große Chance für Zentrumsstraßen ergibt sich durch die Aufhebung seitlicher Parkflächen. Dieser Raum könnte für verschiedene anderweitige Nutzungen verwendet werden.

Der Fußverkehr spielt auch bei multimodalen Verkehrshubs eine zentrale Rolle: Hier sind flächige Fußgängerströme notwendig, um die straff getakteten ÖV-Angebote miteinander zu verbinden. Mit der noch stärkeren Auslastung durch ÖIV-Dienste wird die Situation noch komplexer und die Verkehrsströme nicht mehr klar ablesbar. Die Anlagen sind insbesondere für sensorisch beeinträchtigte Menschen schwierig zu begehen, wenn nicht mehr genügend sichere und rasche Querungsmöglichkeiten geboten werden. Die Folge ist eine Einschränkung der Funktionalität dieser für eine nahtlose Mobilität wichtigsten Anlagen bzw. stellen sich erhöhte Anforderungen an die künftige Gestaltung der Verkehrs- und Aufenthaltsflächen sowie der Einund Aussteigekanten.

In Wohnquartieren kann mit automatisierten Fahrzeugen die Fahrgeschwindigkeit reduziert und die Einhaltung sichergestellt werden, was die Sicherheit für die anderen Straßennutzenden erhöht. Kritisch beurteilt wird die Möglichkeit eines „fahrerlosen Abgeholtwerdens“ von Personen oder Waren, welche zu einer Verkehrszunahme und zu einer sicherheitskritischen 
Verstellung des öffentlichen Raums führen kann. In diesem Zusammenhang stellt sich die Frage, ob Bewilligungen für das „Abgeholtwerden“ erteilt werden müssen oder ob Leerfahrten in solchen Räumen grundsätzlich zu vermeiden sind.

Auf Hauptverkehrsstraßen ist noch unklar, wie in den langen Übergangszeiträumen mit automatisierten und nicht automatisierten Fahrzeugen sowie dem Fuß- und Radverkehr umgegangen werden soll. Es sind Wege zu finden, wie diese in die optimierten und adaptiven Verkehrsführungen, beispielsweise bei Knotensteuerungen, effizient integriert werden können. Dabei ist insbesondere die dafür benötigte (digitale) Kommunikation zwischen den verschiedenen Verkehrsteilnehmenden eine große Herausforderung.

Insbesondere in Gewerbegebieten besteht bei der Ausrichtung der Straßen auf einen automatisierten (Güter-)Verkehr das Risiko, dass der Fuß- und Radverkehr beeinträchtigt wird: Sie sollen den Wirtschaftsverkehr möglichst nicht „stören“. In letzter Konsequenz kann dies dazu führen, dass die Verkehrsträger klar getrennt werden und die verkehrliche Durchlässigkeit eingeschränkt oder ganz aufgehoben wird. Gerade größere Gewerbeareale, die sich einfach abtrennen lassen, könnten zu „verbotenen Städten“ werden. Auch Gewerbegebiete müssen jedoch für Pendelnde sowie Besucherlnnen sicher und mit guten Verbindungen für alle Verkehrsmittel erschlossen werden.

\section{Güterverkehr}

Der Güterverkehr trägt zur Verkehrszunahme in Städten und Agglomerationen mit bei. Sowohl im Bereich von Business-to-Consumer als auch Business-to-Business ist von einer Zunahme der Anzahl an Sendungen sowie von einer Verkleinerung der durchschnittlichen Sendungsgrößen auszugehen. Die Einführung von automatisierten Güterverkehrsfahrzeugen dürfte den Trend zu Mehrverkehr zusätzlich verstärken. Dies ist unter anderem abhängig davon, ob Warenströme gebündelt werden und Fahrzeuge auf der Hin- und Rückfahrt ausgelastet sind. Sobald Güterverkehrsfahrzeuge ohne Fahrerln möglich sind, hat dies auch zur Folge, dass für den Warenumschlag klar definierte Umschlagflächen benötigt werden und bei Bedarf zusätzlich technische Umschlagmittel verfügbar sein müssen. Wenn keine Fahrerlnnen mehr im Fahrzeug sind, werden mehr Aufgaben auf die VersenderInnen oder Empfängerlnnen übertragen oder anderweitige (automatisierte) Systeme wie Paketroboter oder Paketstationen nötig.

Chancen für das automatisierte Fahren bestehen in Gewerbegebieten, die sich aus verschiedenen Gründen als „Testräume“ für automatisiertes Fahren eignen: Zum einen gestaltet sich der Straßenraum häufig weniger vielfältig, mit weniger Fuß- und Radverkehr, häufig liegen sie zudem nahe an Autobahnanschlüssen und benötigen damit nur kurze automatisierte Fahrten auf untergeordneten Straßen. Auch die geringen Geschwindigkeiten erleichtern das Navigieren und Steuern. Zum anderen besteht in Gewerbegebieten auch ein ökonomischer Druck hin zu automatisiertem Fahren, da die Betriebe unmittelbar von Effizienz- und Produktivitätsgewinnen durch neue Logistikkonzepte profitieren können.

Städtebauliche Bedenken oder gestalterische Ansprüche, was die Ausstattung mit spezifischer Infrastruktur oder Straßenmarkierungen betrifft, dürften geringer sein als etwa bei Zentrumsstraßen. Gewerbegebiete eignen sich in der Feinerschließung auch für automatisierte Shuttles. Gerade bei umfassenden Transformationen oder Neuentwicklungen von Gewerbearealen können die Straßenräume unmittelbar auf die Bedürfnisse des automatisierten Fahrens bzw. einer entsprechenden Logistik ausgerichtet werden. Die wirtschaftlichen Argumente eines effizienten Güterverkehrs könnten dazu führen, dass die Bedürfnisse der Unternehmen an Erschließung und Gestaltung gegenüber anderen Ansprüchen an den Straßenraum, etwa durch Fuß- und Radverkehr, stärker gewichtet werden. Im Bereich Güterlogistik bleibt ungeklärt, wo und wer für die neuen automatisierten Umschlagsplätze zuständig und verantwortlich sein wird. 
Die kommerziellen Nutzungen in den Innenstädten bedingen zu unterschiedlichen Tageszeiten Anlieferungen für Verkaufsgeschäfte, Express-, Kurier- und Postdienstleistungen sowie eine Versorgung von Hotels und Restaurants. Entsprechend hoch sind das Aufkommen des Güterund Logistikverkehrs sowie der Bedarf an Güterumschlags- und Haltepunkten.

Die Wirkungen in den Wohnquartieren werden wesentlich davon abhängen, welche Geschäftsmodelle sich auf der letzten Meile etablieren werden, etwa mittels Paketstationen, automatisch zugestellten „Paketfachboxen“ mit Selbstbedienungsfunktion oder gar Lieferrobotern.

\subsection{RÄUMLICHE WIRKUNGEN MIT BLICK AUF DIE DREI SZENARIEN}

Abbildung 6 zeigt im Überblick, welche Mobilitätsräume in Städten und Agglomerationen von Veränderungen in den einzelnen Verkehrsarten besonders betroffen sein dürften, ob positiv oder negativ.

Abbildung 6: Die Mobilitätsräume und deren Ausmaß der Veränderungen in den Verkehrsarten

\begin{tabular}{lccccc}
\hline & $\begin{array}{c}\text { Fließender } \\
\text { Verkehr }\end{array}$ & $\begin{array}{c}\text { Ruhender } \\
\text { Verkehr }\end{array}$ & ÖV/ÖIV & $\begin{array}{c}\text { Fuß- und } \\
\text { Radverkehr }\end{array}$ & $\begin{array}{c}\text { Güterver- } \\
\text { kehr }\end{array}$ \\
\hline $\begin{array}{l}\text { Hauptverkehrs- } \\
\text { straße }\end{array}$ & 0 & 0 & & \\
\hline $\begin{array}{l}\text { Zentrums- } \\
\text { straße }\end{array}$ & 0 & 0 & & \\
\hline $\begin{array}{l}\text { Quartiers- } \\
\text { straße }\end{array}$ & 0 & 0 & & \\
\hline $\begin{array}{l}\text { Gewerbe- } \\
\text { gebiet }\end{array}$ & 0 & 0 & & \\
\hline $\begin{array}{l}\text { Multimodaler } \\
\text { Verkehrshub }\end{array}$ & & & & \\
\hline
\end{tabular}

Quelle: EBP/BaslerFonds/Städteverband und weitere Partner (2018d)

Anhand der Abbildung 6 wird eine räumlich differenzierte Betroffenheit innerhalb der Städte und Agglomerationen erkennbar. Insgesamt am geringsten betroffen sind Hauptverkehrsstraßen und Gewerbegebiete. Diese Mobilitätsräume weisen aufgrund ihrer Funktionen und Nutzungen die tiefste Komplexität auf. In Zentrumsräumen mit ihrer für die Stadtentwicklung bedeutsamen Funktions- und Nutzungsdichte hingegen überlagern sich verschiedene Wirkungen. Dabei ist jedoch darauf hinzuweisen, dass sich die Funktionsfähigkeit eines städtischen Gesamtverkehrssystems aus dem Zusammenspiel aller Verkehrsträger sowie aller Teilräume ergibt.

Die technologischen Entwicklungen und die Beurteilung der möglichen Auswirkungen in den Städten und Agglomerationen sind heute noch mit beträchtlichen Unsicherheiten behaftet. Zudem ist es nicht möglich, die räumlichen Auswirkungen des Einsatzes automatisierter Fahrzeuge isoliert zu betrachten, denn die zukünftige Entwicklung der städtischen Verkehrssysteme wird neben dem automatisierten Fahren von zahlreichen weiteren verkehrlichen, gesellschaftlichen und wirtschaftlichen Faktoren beeinflusst. Außerdem werden die Städte und Agglomerationen noch jahrzehntelang durch ein Nebeneinander von Fahrzeugen mit unterschiedlichen Automatisierungsgraden sowie langfristig auch mit nichtautomatisierten Fahrzeugen und weiteren Verkehrsteilnehmenden geprägt sein. 
Der zusammenfassende Blick auf diese kleinräumigen Auswirkungen ermöglicht auch eine kurze Einschätzung und Beurteilung der drei Szenarien der TA-SWISS-Studie aus verkehrs- und raumpolitischer Perspektive.

In Szenario 1, mit einer stark individuellen Nutzung in allen Räumen, werden zwei große Herausforderungen erkennbar: eine stark steigende Verkehrsbelastung bzw. eine sinkende Aufenthalts- und Lebensqualität in städtischen Räumen sowie eine verstärkte Tendenz zur Zersiedlung in ländlich geprägten Räumen. Inwieweit sich Attraktivitätsverschiebungen zwischen den Räumen aufgrund des automatisierten Fahrens in der großräumigen Raumstruktur niederschlagen, wird maßgeblich vom regulatorischen Umgang mit dem automatisierten Fahren sowie der verkehrs- und raumplanerischen Steuerung abhängen. Hier bietet das automatisierte Fahren die Chancen, intermodale Mobilitätsketten zu optimieren, die Verknüpfung der verschiedenen Verkehrsmittel und -träger zu verbessern und bisher mobilitätseingeschränkte Bevölkerungsgruppen zu integrieren. Insgesamt steht das Szenario jedoch im Widerspruch zur schweizerischen Klima-, Verkehrs- und Raumentwicklungspolitik.

In Szenario 3, mit einer schweizweit stark kollektiv geprägten Nutzung, stellen sich Fragen nach der Rentabilität bzw. der Finanzierbarkeit flächendeckender kollektiver Angebote. Hier sind neben verkehrspolitischen auch staats- und regionalpolitische Überlegungen relevant. Welche Grundversorgung, in der Schweiz auch „Service Public“ genannt, will sich die Schweiz als föderalistisches Land in welcher Qualität leisten? Und von wem soll sie finanziert werden? Aus einer heutigen Betrachtung, und mit Blick auf die Diskussionen der letzten Jahrzehnte, erscheint die Entwicklung und Finanzierung eines solchen regulatorischen Rahmens nicht realistisch. Gezielt eingesetzte kollektive Angebote können aber auch in peripheren Räumen die Versorgungsqualität verbessern oder zumindest dazu beitragen, sie zu erhalten.

Insbesondere aus Sicht der Städte und Agglomerationen dürfte Szenario 2 ganz klar bevorzugt sein bzw. als zwingend erscheinen. Wie anlässlich der Diskussionen im Rahmen des Projektes ersichtlich wurde, überwiegen bei den VertreterInnen der Städte und Agglomerationen skeptische Einschätzungen zu den möglichen Wirkungen. Aus Sicht der Städte ist die langfristige Wirkung wesentlich davon abhängig, ob es gelingt, die Vorteile des automatisierten Fahrens überwiegend zur Stärkung des kollektiven Verkehrs zu nutzen. Von einer (relativen) Attraktivitätssteigerung des MIV werden hingegen überwiegend negative Folgen erwartet. Neben einer Konkurrenzierung des ÖV befürchten die Städte und Agglomerationen auch eine Beeinträchtigung des Fuß- und Radverkehrs und der Gestaltung der öffentlichen Räume. Hier besteht gleichzeitig auch die große Chance, allfällige Effizienzsteigerungen beim motorisierten Verkehr zugunsten des Fuß- und Radverkehrs zu nutzen.

\section{DER BEDARF UND DIE ANSÄTZE EINES REGULIERUNGSRAHMENS FÜR DIE SCHWEIZ}

\subsection{POLITISCHER HANDLUNGSBEDARF}

Aufbauend auf den Erkenntnissen aus den Szenarien wurden im Rahmen der TA-SWISS-Studie in einem Expertenworkshop Regulierungsoptionen erarbeitet, mit welchen sich absehbare Entwicklungen für das automatisierte Fahren in der Schweiz politisch steuern ließen. Die ExpertInnen waren sich darin einig, dass das automatisierte Fahren kommt und dass es 
Regulierung schon heute braucht und nicht erst in zwanzig oder dreißig Jahren. Ein „Laisserfaire" würde zu starken Verkehrsverlagerungen vom kollektiven zum individuellen Verkehr führen. Eine solche Entwicklung könnte - aller Effizienzgewinne durch das automatisierte Fahren zum Trotz - mehr Stau und/oder Forderungen nach einem Ausbau der Infrastruktur hervorrufen.

Welche Wirkungen mit einer solchen Regulierung erreicht werden sollen und welches Szenario anzustreben ist, war und bleibt unter den ExpertInnen aber umstritten. Diese Frage hat eine normative Komponente, welche letztlich auf eine gesellschaftliche Zielvorgabe abstellen muss; solche Zielvorgaben fehlen aber zurzeit in der Schweiz oder widersprechen sich. Zudem ist nicht zu erwarten, dass sie in der nächsten Zeit in der notwendigen Klarheit zur Verfügung stehen werden. Die damit verbundenen Fragen sind komplex und gleichzeitig in vielem noch zu vage, um einen gesellschaftlichen und politischen Konsens herbeiführen zu können.

Die im Expertenworkshop entwickelten Regulierungsoptionen wurden in einem nächsten Arbeitsschritt mit StakeholderInnen aus Politik, Verwaltung, Wirtschaft und Verbänden diskutiert. Auch in diesem Kreis wurde der Stellenwert des automatisierten Fahrens für das Mobilitätssystem allgemein als hoch eingeschätzt. Diese Einschätzung beruht vor allem auf erwarteten Effizienzgewinnen. Es wurde aber ebenfalls deutlich, dass automatisiertes Fahren auch einen Beitrag zur Nachhaltigkeit des Verkehrs allgemein leisten kann und leisten soll.

Dass es schon heute wichtig und dringlich ist, einen gesellschaftlichen und politischen Diskurs über Regulierungsoptionen zu führen, wurde allgemein befürwortet. Die Diskussion mit den StakeholderInnen zeigte im Gegensatz zu den ExpertInnen aber, dass in wichtigen Aspekten wohl durchaus schon ein impliziter Konsens besteht. So zum Beispiel darin, dass das Grundprinzip des schweizerischen Mobilitätssystems mit der bedeutenden Rolle des ÖV erhaltenswert sei und die Schweiz in diesem Bereich auch eine Vorreiterrolle bei der weiteren Entwicklung der automatisierten Mobilität einnehmen könne und solle. Auch Zufußgehende und Radfahrende sollen weiterhin Platz im Verkehrsraum finden, insbesondere in dicht besiedelten Gebieten. Zudem soll das Gesamtverkehrssystem umwelt- und klimaverträglich sein.

\subsection{ROLLE DES STAATES UND HANDLUNGSOPTIONEN}

Zwei Maßnahmen sind zwingend, um das automatisierte Fahren ab Stufe 3 in der Schweiz überhaupt erst zulassen zu können und in diesem Sinne eine Voraussetzung für weitere Handlungen zu schaffen:

Es sind die mit dem Ausland abgestimmten Voraussetzungen für die Zulassung von bedingt automatisierten sowie hoch- und vollautomatisierten Fahrzeugen in der Schweiz zu schaffen, sodass auf dem Markt verfügbare Fahrzeuge ab Stufe 3 für den Personen- und Güterverkehr auch eingesetzt werden können. Dies betrifft v. a. die Zulassung der automatisierten Systeme sowie die Regelung der Haftungsfrage. Zusätzlich müssen in Abstimmung mit den Nachbarländern Sicherheitsstandards, beispielsweise bezüglich der Übergabezeiten, festgelegt werden. Weitere, schweizspezifische Auflagen für die Vernetzung und den Datenaustausch oder allfällige Vorgaben für energieeffiziente Antriebe müssen frühzeitig geprüft werden.

Es sind Aus- und Weiterbildungen zum Umgang mit (voll)automatisierten Fahrzeugen vorzusehen, etwa ein „Führerschein light“ resp. ein Fähigkeitsausweis zur Beaufsichtigung hochautomatisierter Fahrzeuge der Stufen 3 und 4. 
Über diese beiden zwingenden Maßnahmen hinaus können zwei Grundrichtungen des staatlichen Handelns bei der Einführung von Automatisierung und Vernetzung im Verkehr in der Schweiz unterschieden werden:

1. „Enabler“: Der Staat kann eine gestaltende, aber eher zurückhaltende Rolle einnehmen, die sich darauf beschränkt, liberale Rahmenbedingungen zu setzen, sodass Marktinnovationen und Entwicklungen von privaten und öffentlichen Akteuren möglich werden.

2. „Leader“: Ausgehend von klaren politischen Zielsetzungen kann der „starke Staat“ aktiv Regulierungen, die entweder die Entwicklung von Automatisierung und Vernetzung im Verkehr einschränken resp. verzögern oder im Gegenteil auch aktiv fördern können, durchsetzen.

In Bezug auf diese zwei möglichen Rollen des Staates konnten keine mehrheitsfähigen Präferenzen der Studienbeteiligten ausgemacht werden. Je nach politischer Grundhaltung oder auch je nach Teilaspekt der Automatisierung und Vernetzung vertraten die Expertlnnen und StakeholderInnen unterschiedliche Standpunkte. Entsprechend werden aus heutiger Sicht beide übergeordneten Grundrichtungen als ein für die Schweiz gangbarer Weg betrachtet. Um adäquat auf diese Breite und die Unsicherheit in Bezug auf die technische, rechtliche und zeitliche Entwicklung von automatisierten und vernetzten Fahrzeugen zu reagieren, werden die konkreten Handlungsoptionen in Bezug zu den zwei übergeordneten Grundrichtungen gesetzt.

Für die gestaltende, aber zurückhaltende Enabler-Rolle werden zusätzlich zu den zwingenden Maßnahmen folgende Handlungsoptionen empfohlen:

- Es sind gesellschaftliche und politische Diskussionen zur Nutzung der von Fahrzeugen produzierten Daten in Bezug auf ethische und Datenschutzaspekte zu führen. Chancen und Risiken der Nutzung von Daten sollten offen mit der Zivilgesellschaft diskutiert werden.

- Es ist frühzeitig und staatsebenenübergreifend eine Position in der Datenpolitik zu formulieren, welche die Interessen der öffentlichen Hand festhält. Dazu gehört die Festlegung der für ihre Aufgabe notwendigen Daten sowie der Datenherrschaft und Zugriffsrechte. Eine Abstimmung mit Smart City-Strategien und „Open Governement Data“-Prinzipien ist zentral. In Ergänzung zu den international festgelegten Standards für den Datenaustausch kann die Schweiz darüberhinausgehende Qualitätsstandards und Vorgaben für die Metadaten zu den ausgetauschten Daten formulieren.

- Das Schweizerische Personenbeförderungsgesetz und die dazugehörige Verordnung sind anzupassen. Einerseits sind im bisher stark regulierten Personentransport neue Anbieter zuzulassen, andererseits sind für den kollektiven Verkehr ergänzend zum bisherigen ÖVVerständnis flexiblere Angebote (z. B. das Erteilen von Konzessionen, die anstelle einer Fahrplanpflicht oder des Linienverkehrs flexiblere, aber in Bezug auf das Minimalangebot definierte Gebietserschließungen vorsehen - sofern die Flächeneffizienz des Verkehrssystems nicht darunter leidet) oder bedingte Erleichterungen bei der Barrierefreiheit zu ermöglichen.

- Die Bewilligungspraxis und die Vorgaben an konzessionierte Transportunternehmen sind zu lockern, damit diese einfacher forschen und entwickeln können. Die betrieblichen, straBenverkehrsrechtlichen und versicherungstechnischen Rahmenbedingungen sind so zu gestalten, dass eigenwirtschaftliche und verursachergerecht finanzierte öffentliche und private Fahrdienste möglich sind. 
- Es sind attraktive Bedingungen für den Aufbau eines leistungsfähigen Mobilfunknetzes zu schaffen. Im Hinblick auf die Situation in der Schweiz mit mehreren privaten Betreibern bisheriger Mobilfunknetze könnte auch die Option eines staatlichen Netzes im Sinn eines „utility networks“, auf dem Private Dienstleistungen anbieten können, interessant und entsprechend zu prüfen sein.

- Es ist eine Datenplattform mit „bedingter Open Data“2 einzurichten, an der sich alle Akteure mit eigenen Daten beteiligen und anderweitige Daten beziehen können (ASTRA 2018). Dort sollen zudem topographische und verkehrliche Basisdaten, Ereignisdaten, Messdaten und aggregierte Sensordaten verfügbar sein. Dazu gilt es unter anderem, technische Systeme für durchgehende digitale und automatisierbare Workflows weiterzuentwickeln und entstehenden internationalen Standards anzupassen.

Auf Basis von klaren politischen Zielen kann der Staat eine Leader-Rolle einnehmen. Dazu sollten - ergänzend zu den bisher erwähnten Empfehlungen - folgende Maßnahmen und Instrumente ergriffen werden:

- Es ist ein Dialog über die erwünschte Mobilität der Zukunft auf allen staatlichen Ebenen anzustoßen. Daran sollen sich sowohl Politik und Wissenschaft als auch die Zivilgesellschaft und die Wirtschaft beteiligen. Dazu gehört auch der Diskurs über die Sicherheit und Wünschbarkeit von technischen Systemen.

- Es sind Zielvorgaben für die künftige Mobilität in der Schweiz zu erarbeiten. Dabei sind die Bedürfnisse des Bundes, der Städte, der Kantone, der Gesellschaft und Wirtschaft gleichermaßen sowie der Personen- und Güterverkehr zu berücksichtigen. Auch Zielkonflikte auf nationaler Ebene zu Vorgaben aus anderen Politikbereichen sind zu thematisieren.

- Es sind in Zusammenarbeit aller Staatsebenen und unter Einbezug von Wirtschaft und Zivilgesellschaft Ideen und Vorschläge zu entwickeln, wie das automatisierte und vernetzte Fahren in Städten und Agglomerationen v. a. kollektive Formen der Mobilität und des Transports stärken kann. Dies bedingt voraussichtlich Anreize für den ÖIV und neue Rahmenbedingungen für den ÖV, allenfalls aber auch Restriktionen für den individuellen automatisierten Verkehr mit privaten Fahrzeugen. Zu thematisieren sind sowohl marktwirtschaftliche Instrumente, Auflagen in Konzessionen oder Zulassungen (z. B. keine Leerfahrten) oder Information und Persuasion.

- $\quad$ Es ist eine hoheitliche Verkehrssteuerung vorzusehen. Dazu ist zwischen Städten, Kantonen und dem Bund einerseits zu diskutieren, wer für diese Verkehrssteuerung zuständig ist und wie allfällige Schnittstellen behandelt werden. Andererseits muss unter Einbezug von Wissenschaft und den Transportunternehmen festgelegt werden, über welche Kennwerte die Verkehrssteuerung idealerweise erfolgt (Besetzungsgrad, Mindesttransportlänge, Fahrleistung, Zielwahl, Parkplätze, Haltestellen etc.).

2 Im Datenverbund sind nach den Prinzipien von „bedingter Open Data“ die Daten frei zugänglich: Jeder kann sie frei beziehen, muss aber selbst Daten zurückliefern, sofern mit den bezogenen Daten ein Geschäftsmodell betrieben wird. Die so erweiterten Daten stehen wieder allen zur Verfügung und können für neue Anwendungen genutzt werden. Davon ausgenommen sind „veredelte Daten“, also Informationen, die weiterhin auf dem Markt gehandelt werden können. Wo die Grenze zwischen Daten und Informationen liegen, müssen die Beteiligten gemeinsam aushandeln (ASTRA 2018). 
Zusammenfassend lässt sich erkennen, dass der größte Regulierungsbedarf folgerichtig bei der Einnahme einer Leader-Rolle anfällt. Es braucht Zielbilder und Strategien, um als starker Staat voranzugehen und die Entwicklung von Beginn an in die gewünschte Richtung zu lenken. Explizit formulierte Ziele und Strategien erfordern aber einen politischen Aushandlungsprozess, der in der Schweiz über längere Zeit andauern kann. Wenn die Enabler-Rolle des Staates im Zentrum stehen soll, dann lassen sich solche expliziten Zielbilder und Strategien auch iterativ entwickeln. Dies geht über eine rein reaktive Politik hinaus, stellt jedoch weniger Anforderungen an den Zieldiskurs, der für eine proaktive Politik im Sinne einer Leader-Rolle notwendig ist.

\subsection{SPEZIFISCHE HANDLUNGSOPTIONEN FÜR STÄDTE UND AGGLOMERATIONEN}

Die Herausforderung für die Städte und Agglomerationen ist und bleibt, einen siedlungs- und klimaverträglichen Verkehr zu gestalten. Handlungsoptionen bezüglich des Einsatzes automatisierter Fahrzeuge sind deshalb in eine verkehrs-, raum- und städteplanerische Gesamtbetrachtung einzubetten. Die BaslerFonds-Studie formuliert zehn strategische Stoßrichtungen mit möglichen Maßnahmen für Städte und Agglomerationen.

- Mobilität gesamtheitlich und stadtverträglich planen: Es gilt, sich Gedanken darüber zu machen, wie automatisiertes Fahren zur erwünschten klima- und stadtverträglichen Mobilität beitragen kann.

- Möglichkeiten testen, Erfahrungen austauschen und Wissen erweitern: Mit Testversuchen und Pilotprojekten können Erfahrungen gesammelt und Erkenntnisse zu verkehrlichen und räumlichen Wirkungen gewonnen werden. Ein Austausch zwischen den Städten sowie mit Bund und Kantonen ermöglicht die Nutzung von Synergien und verhilft zu einer schrittweisen Weiterentwicklung des Wissensstandes.

- Bedürfnisse und Anliegen der Städte und Agglomerationen einbringen: Städte und Agglomerationen sollten die Entwicklungen und Anwendungen des automatisierten Fahrens aktiv verfolgen, Wissen und Erfahrungen austauschen und eine gemeinsame Meinungsbildung pflegen. Auf dieser Basis können sie ihre Bedürfnisse und Anliegen gegenüber dem Bund und den Kantonen einbringen.

- Diskurs pflegen und Sensibilisierung erhöhen: Ein öffentlicher Diskurs auf allen Ebenen erhöht die Sensibilisierung für das automatisierte Fahren und ermöglicht eine breit abgestützte Debatte zu den verkehrlichen und stadträumlichen Einflüssen und Wirkungen.

- Mehrverkehr begrenzen: Der durch die neuen Mobilitätsangebote und Leerfahrten induzierte Mehrverkehr muss mit geeigneten Maßnahmen minimiert resp. gezielt gelenkt werden.

- Kollektive Transportangebote stärken: Als flächeneffizienteste Form gilt es, den ÖV und ÖIV weiterhin attraktiv, kostengünstig und effizient auszugestalten und den Modalsplit zugunsten der nachhaltigen Mobilitätsformen mit attraktiven Angeboten weiter zu beeinflussen.

- Verkehrsströme intelligent lenken und steuern: Mit einer hohen Durchdringung von automatisierten Fahrzeugen und einer ausgebauten Vernetzung lässt sich der Gesamtverkehr intelligenter lenken und steuern. Nachfragespitzen können räumlich und zeitlich besser beeinflusst werden. 
- Öffentliche und private Flächennutzung optimieren: Freiwerdende Parkflächen können zur Steigerung der Attraktivität des Stadtraums genutzt werden. Ergänzend sind Konzepte zum Parken, zu Haltezonen und Umschlagsflächen für den automatisierten Personen- und Güterverkehr zu entwickeln.

- Sicherheit für alle Verkehrsteilnehmenden gewährleisten: Mit geeigneten Maßnahmen können Sicherheitsrisiken im Nebeneinander der Verkehrsteilnehmenden sowie in der Übergangszeit wie auch bei Vollautomatisierung reduziert werden.

- Citylogistik siedlungsverträglich mitgestalten: Städte und Agglomerationen können privatwirtschaftliche Bemühungen für effiziente Warenlieferungskonzepte unterstützen und siedlungsverträglich regulieren.

\section{DIE STEUERUNG VON MOBILITÄT UND RAUMENTWICKLUNG NICHT AUS DER HAND GEBEN}

Anhand der drei Szenarien aus der TA-SWISS-Studie wurde untersucht, in welche Richtungen die Entwicklung resp. Einführung von automatisiertem Fahren in der Schweiz gehen könnte. Ziel der Studie war nicht, sich für ein bestimmtes Szenario auszusprechen, sondern Handlungsoptionen zu formulieren, die entweder szenarienübergreifend Sinn machen oder dann aufzeigen, mit welcher Rolle des Staates welche Regulierungen verbunden wären.

Zwischen den Beteiligten an der BaslerFonds-Studie hingegen wurde deutlich, in welche Richtung die Entwicklung in Städten und Agglomerationen gehen sollte, damit den Menschen auch in Zukunft lebenswerte (Straßen-)Räume geboten werden können. Gewünscht sind kollektive Mobilitätsangebote in städtischen Räumen, die in Szenario 2 der TA-SWISS-Studie aufgezeigt wurden. Entsprechend ist es aus Sicht der Städte und Agglomerationen für eine nachhaltige Mobilität nicht ausreichend, dass sich der Staat nur auf die Enabler-Rolle beschränkt und die Entwicklung dem Markt überlässt.

Das Einnehmen der Leader-Rolle erfordert aber zuerst klare Zielvorgaben für die künftige Mobilität. Unter Berücksichtigung des Politikzyklus ${ }^{3}$ (Abb. 7) sind solche Mobilitätsstrategien im Idealfall vorgelagert zu regulatorischen Eingriffen zu definieren resp. zwischen Bund, Städten, Kantonen sowie der Gesellschaft und Wirtschaft auszuhandeln. Dabei können und sollen auch - je nach Raumtyp - unterschiedliche Stoßrichtungen Platz finden resp. diese Leader-Rolle mit unterschiedlicher Intensität wahrgenommen werden. Dabei sind aber Zielkonflikte zu Vorgaben aus anderen Politikbereichen zu lösen.

Erst wenn Klarheit und Einigkeit zu diesen Zielen besteht, sollen auf allen staatlichen Ebenen die im vorliegenden Beitrag beschriebenen Instrumente und Regulatorien zu den politischen Maßnahmenbereichen geprüft und festgelegt werden, wobei es sich hier nicht um einen rein linearen Prozess handelt - Zielsetzungen und Maßnahmen können und sollen auch iterativ entwickelt werden. Gerade wenn die Enabler-Rolle des Staates im Zentrum stehen soll, dann

3 http://politischebildung.ch/fuer-lehrpersonen/didaktik-und-methoden/politikzyklus, www.staatsfragen.de/tag/politikzyklus/. 
lassen sich solche expliziten Zielbilder und Strategien auch iterativ entwickeln. Dies geht über eine rein reaktive Politik hinaus, stellt jedoch weniger Anforderungen an den Zieldiskurs, der für eine proaktive Politik im Sinne einer Leader-Rolle notwendig ist.

Zudem müssen die internationalen Entwicklungen laufend beobachtet werden, um auf allen Staatsebenen angemessen und rasch reagieren zu können. Von besonderem Interesse sind rechtliche Festlegungen der EU, aber auch in anderen führenden Staaten, sowie allgemeine technologische Fortschritte beim automatisierten und vernetzten Fahren. Internationale Erkenntnisse müssen rasch in die Schweizer Politikkreisläufe Eingang finden, um die Handlungsoptionen möglichst wirksam und im Sinne der Mobilitätsstrategien zu evaluieren und bei Bedarf zu revidieren.

Abbildung 7: Politikzyklus für den Einsatz automatisierter Fahrzeuge in der Schweiz

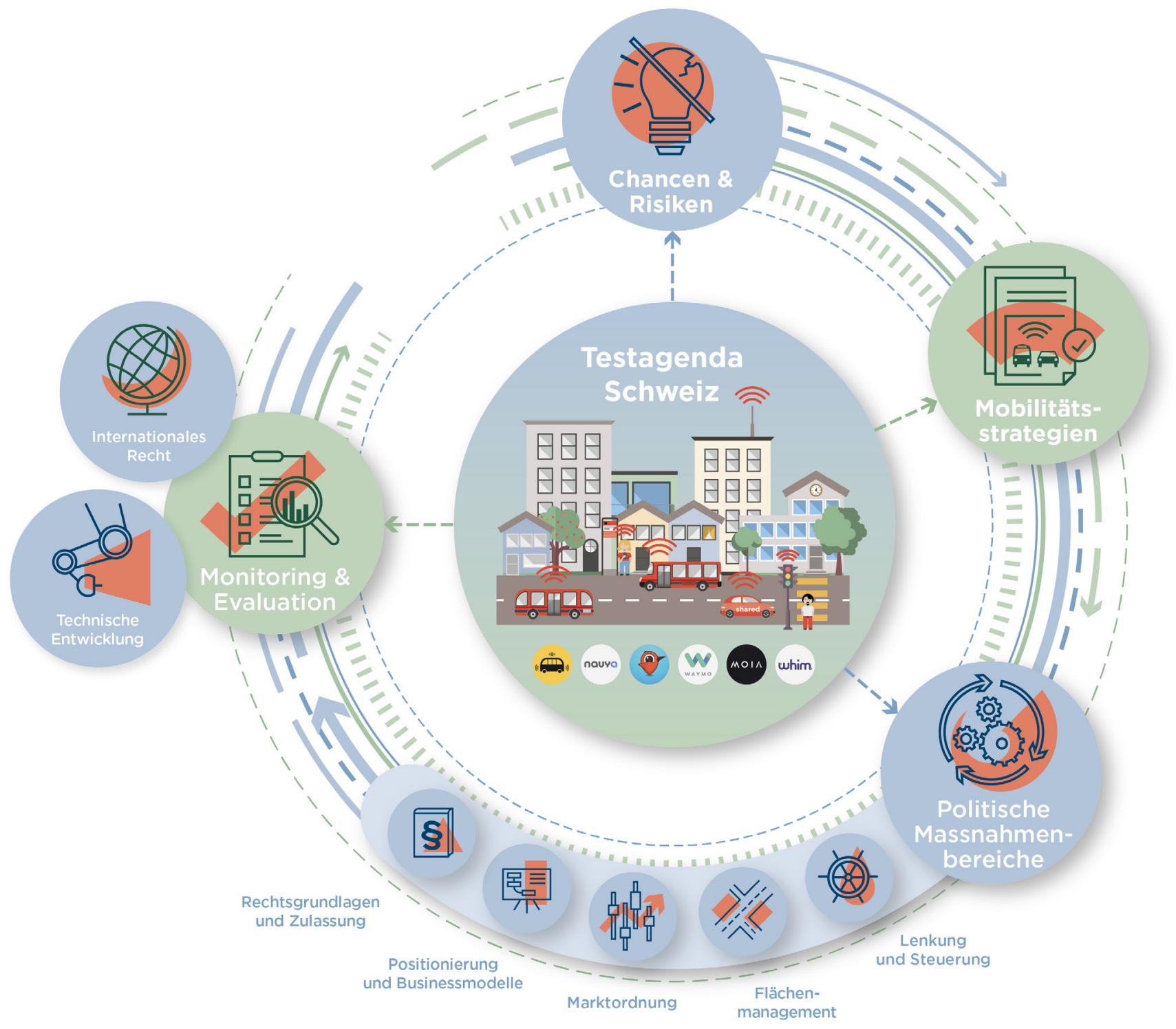

Quelle: EBP/BaslerFonds/Städteverband und weitere Partner (2018g) 
Zudem ist aufzuzeigen, dass mit geeigneten Maßnahmen negative Wirkungen des automatisierten Fahrens erfolgreich vermieden oder zumindest eingeschränkt werden können. Dazu sind von Bund, Kantonen, Städten und Gemeinden gemeinsam und frühzeitig entsprechende Monitoring- und Controllingsysteme zu entwickeln und auf ihre Anwendbarkeit zu prüfen. Die Festlegung der notwendigen Regulatorien für die Beeinflussung der Mobilität und des Verkehrs muss auch vor diesem Hintergrund erfolgen. Falls die gewünschten Wirkungen gering sind oder ganz ausbleiben, muss über eine Überarbeitung der Mobilitätsstrategien, der rechtlichen Grundlagen oder der implementierten Instrumente nachgedacht werden. Auf allen Staatsebenen braucht es die Bereitschaft für entsprechende Korrekturprozesse.

Viele künftige Chancen und Herausforderungen des automatisierten Fahrens sind aus heutiger Sicht nur schwer voraussehbar oder modellierbar. Mit Testanlagen und Pilotprojekten können wichtige Erfahrungen gesammelt und Erkenntnisse zu verkehrlichen wie räumlichen Wirkungen gewonnen werden. Eine wichtige Erkenntnis beider Studien ist denn auch, dass Pilotanwendungen eine zentrale Grundlage für alle vier Schritte des politischen Kreislaufes sind. Aus diesem Grund wird der Politikzyklus in Abbildung 7 mit einer zusätzlichen Dimension versehen: Innerhalb dieser Testagenda laufen im kleineren Rahmen auch die vier Schritte des Kreislaufes ab. Da die Implementierung aber über Sonder- und Ausnahmebewilligungen erfolgt, kann sich dieser Kreislauf schneller bewegen und wichtige Erkenntnisse für die anderen Schritte liefern.

Die Erfahrungen aus diesen Anwendungen sollen einerseits Evidenz schaffen und zielführende Anwendungen für die Schweiz aufzeigen, andererseits soll der Bund die Erkenntnisse auch im Rahmen seiner internationalen Tätigkeit bei rechtlichen Fragen einbringen.

\section{LITERATUR}

ASTRA (Bundesamt für Strassen) 2018. „Bereitstellung und Austausch von Daten für das automatisierte Fahren im Strassenverkehr“, 7.12.2018, Bern. www.astra.admin.ch/dam/astra/de/dokumente/ abteilung_strassennetzeallgemein/bereitstellung-austausch-daten-automatisiertes-fahren.pdf. download.pdf/Bereitstellung\%20und\%20Austausch\%20von\%20Daten\%20f\%C3\%BCr\%20das\%20 automatisierte\%20Fahren\%20im\%20Strassenverkehr.pdf (9.7.2020).

EBP, BaslerFonds, Städteverband und weitere Partner 2017. „Einsatz automatisierter Fahrzeuge im Alltag - Denkbare Anwendungen und Effekte in der Schweiz. Schlussbericht Grundlagenanalyse (Phase A)“, Zürich. www.ebp.ch/sites/default/files/project/uploads/171024_BaslerFonds_aFz_ Phase\%20A_Schlussbericht_de_1_1.pdf (1.7.2020).

EBP, BaslerFonds, Städteverband und weitere Partner 2018a. „Einsatz automatisierter Fahrzeuge im Alltag - Denkbare Anwendungen und Effekte in der Schweiz. Schlussbericht Modul 3a, Verkehrstechnik“", Zürich. www.ebp.ch/sites/default/files/project/uploads/2018-04-04\%20aFn_3a\%20Verkehrstechnik_Schlussbericht_0.pdf (1.7.2020).

EBP, BaslerFonds, Städteverband und weitere Partner 2018b. „Einsatz automatisierter Fahrzeuge im Alltag - Denkbare Anwendungen und Effekte in der Schweiz. Schlussbericht Modul 3b, Daten und IT-Infrastrukturen“", Zürich. www.ebp.ch/sites/default/files/project/uploads/2018-04-05\%20 aFn_3b\%20Daten\%20und\%20Infrastrukturen_Schlussbericht_0.pdf (1.7.2020).

EBP, BaslerFonds, Städteverband und weitere Partner 2018c. „Einsatz automatisierter Fahrzeuge im Alltag - Denkbare Anwendungen und Effekte in der Schweiz. Schlussbericht Modul 3c, Mögliche Angebotsformen im kollektiven Verkehr (ÖV und ÖIV) “', Zürich. www.ebp.ch/sites/default/files/ project/uploads/2018-04-19\%20aFn_3c\%20Mögliche\%20Angebotsformen\%20im\%20kollektiven\%20Verkehr_Schlussbericht_0.pdf (1.7.2020). 
EBP, BaslerFonds, Städteverband und weitere Partner 2018d. „Einsatz automatisierter Fahrzeuge im Alltag - Denkbare Anwendungen und Effekte in der Schweiz. Schlussbericht Modul 3d, Städte und Agglomerationen“", Zürich. www.ebp.ch/sites/default/files/project/uploads/2018-08-30\%20 aFn_3d\%20Städte-Agglomerationen\%20Schlussbericht_1.pdf (1.7.2020).

EBP, BaslerFonds, Städteverband und weitere Partner 2018e. „Einsatz automatisierter Fahrzeuge im Alltag - Denkbare Anwendungen und Effekte in der Schweiz. Schlussbericht Modul 3e, Ressourcen, Umwelt, Klima““, Zürich. www.ebp.ch/sites/default/files/project/uploads/2018-04-09\%20 aFn_3e\%20Ressourcen\%2C\%20Umwelt\%2C\%20Klima_Schlussbericht_0.pdf (1.7.2020).

EBP, BaslerFonds, Städteverband und weitere Partner 2018f. „Einsatz automatisierter Fahrzeuge im Alltag - Denkbare Anwendungen und Effekte in der Schweiz. Schlussbericht Modul 3f, Güterverkehr/ City Logistik (Strasse)““, Zürich. www.ebp.ch/sites/default/files/project/uploads/2018-03-28\%20 aFn_3f\%20Güterverkehr\%20und\%20Citylogistik_Schlussbericht_0.pdf (1.7.2020).

EBP, BaslerFonds, Städteverband und weitere Partner 2018g. „Einsatz automatisierter Fahrzeuge im Alltag - Denkbare Anwendungen und Effekte in der Schweiz. Synthese“, Zürich. www.ebp.ch/sites/ default/files/project/uploads/180905_Synthese_BaFoaFn_def.pdf (1.7.2020).

EBP, Fonds für Verkehrssicherheit 2018. „Automatisiertes Fahren Auswirkungen auf die Strassenverkehrssicherheit. Schlussbericht vom 31. Mai 2018“, Zürich. www.ebp.ch/sites/default/files/project/ uploads/2018-05-31\%20aFn_Verkehrssicherheit_Schlussbericht.pdf (9.7.2020).

Perret, F., T. Arnold, R. Fischer, P. de Haan und U. Haefeli 2020. Automatisiertes Fahren in der Schweiz: Das Steuer aus der Hand geben?, TA-SWISS 71/2020. Zürich: vdf.

Open Access Dieser Beitrag wird unter der Creative Commons Namensnennung 4.0 International Lizenz (http://creativecommons.org/licenses/by/4.0/deed.de) veröffentlicht, welche die Nutzung, Vervielfältigung, Bearbeitung, Verbreitung und Wiedergabe in jeglichem Medium und Format erlaubt, sofern Sie den/die ursprünglichen Autor(en) und die Quelle ordnungsgemäß nennen, einen Link zur Creative Commons Lizenz beifügen und angeben, ob Änderungen vorgenommen wurden.

Die in diesem Beitrag enthaltenen Bilder und sonstiges Drittmaterial unterliegen ebenfalls der genannten Creative Commons Lizenz, sofern sich aus der Abbildungslegende nichts anderes ergibt. Sofern das betreffende Material nicht unter der genannten Creative Commons Lizenz steht und die betreffende Handlung nicht nach gesetzlichen Vorschriften erlaubt ist, ist für die oben aufgeführten Weiterverwendungen des Materials die Einwilligung des jeweiligen Rechteinhabers einzuholen.

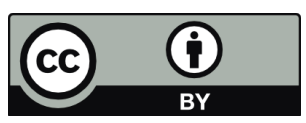

